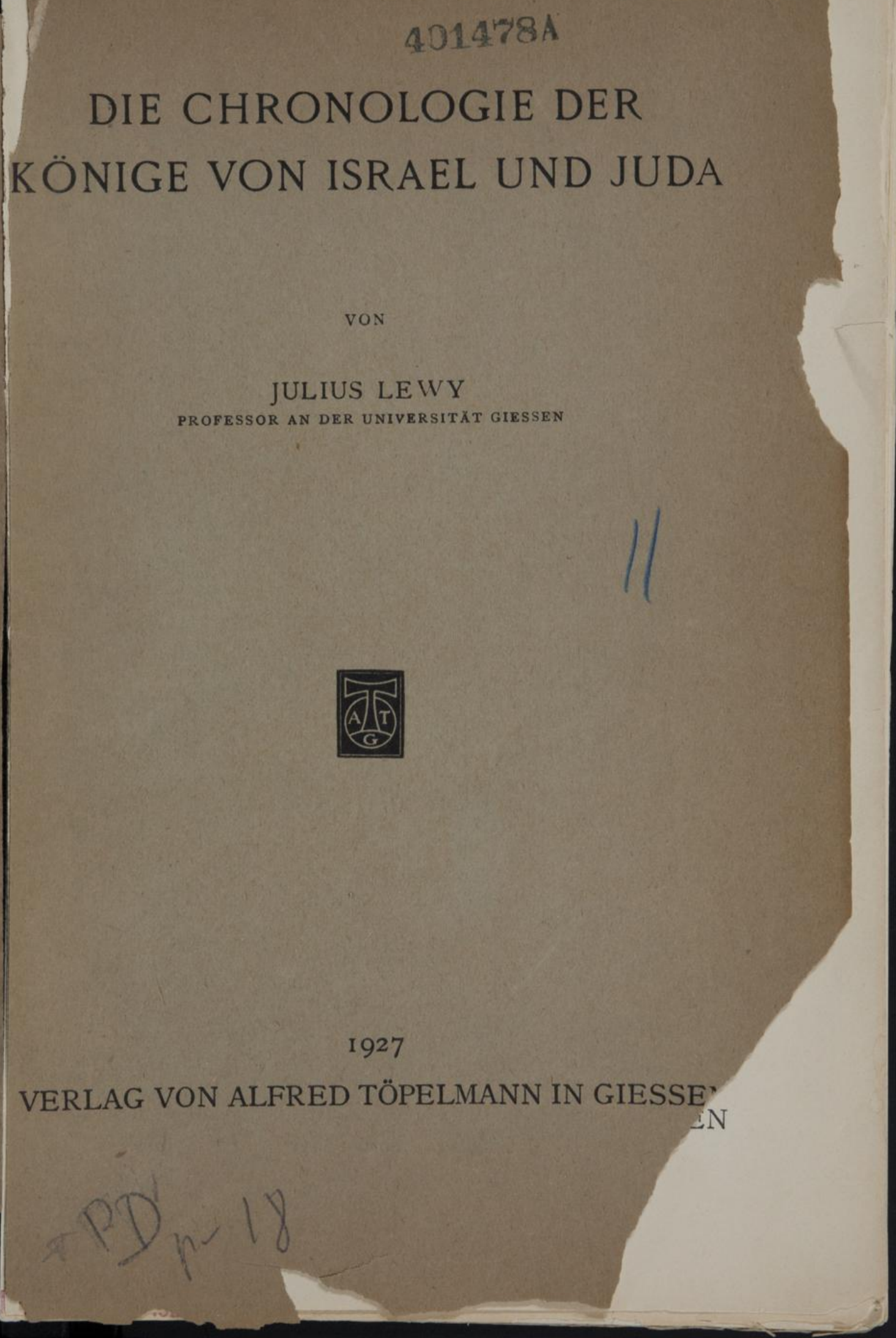




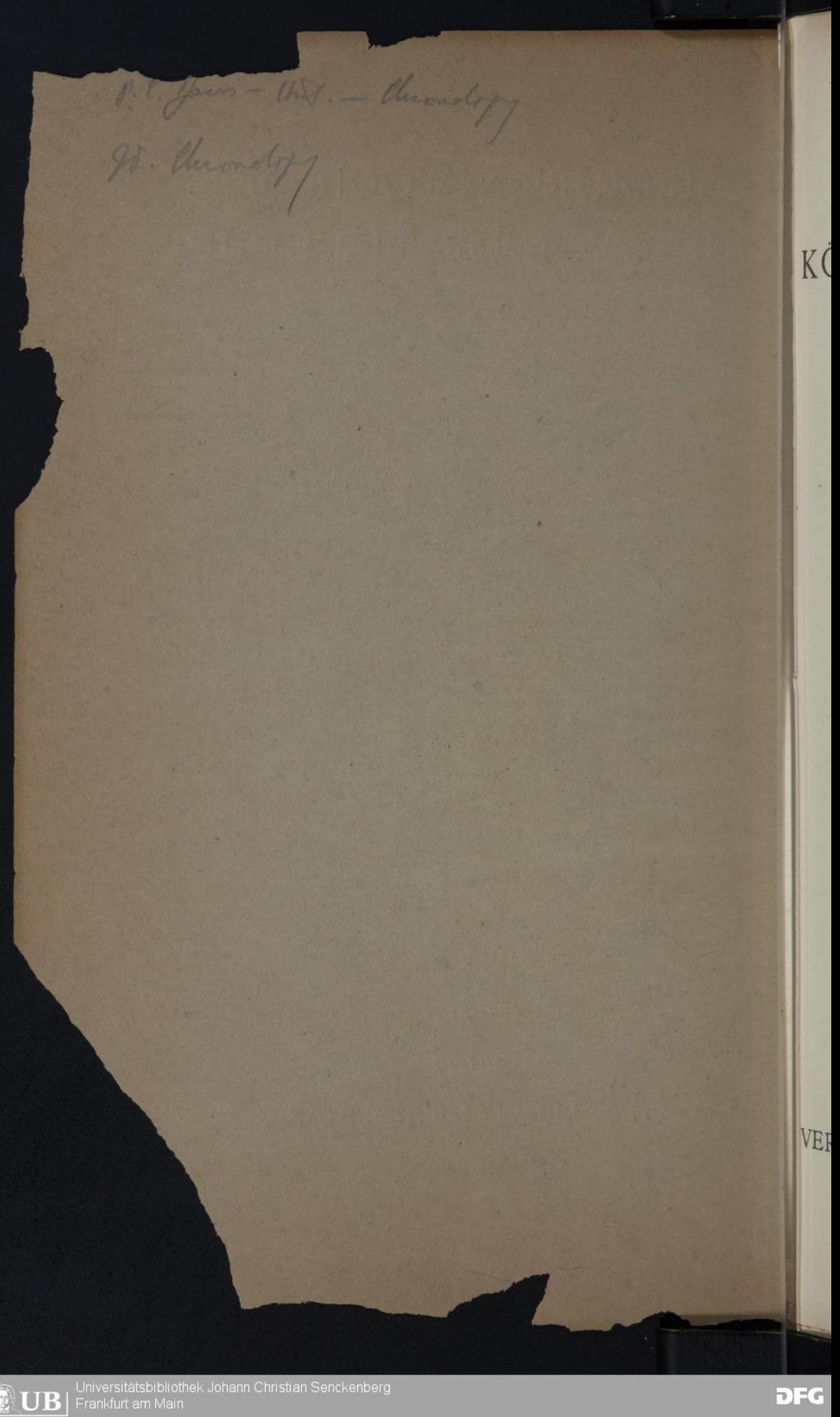




\section{DIE CHRONOLOGIE DER KÖNIGE VON ISRAEL UND JUDA}

JULIUS LEWY

PROFESSOR AN DER UNIVERSITÄT GIESSEN

I 927

VERLAG VON ALFRED TÖPELMANN IN GIESSEN cre 
Alle Rechte, besonders das Recht der Übersetzung in fremde Sprachen, werden vorbehalten.

\section{LEO BAECK IMSTITUTE \\ 051013}


DEM ANDENKEN

AN

PAUL HAUPT

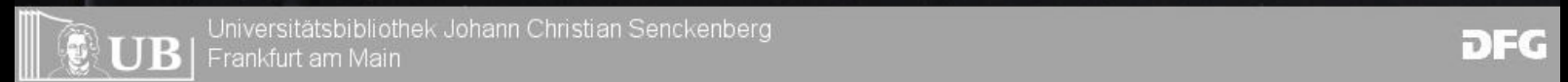



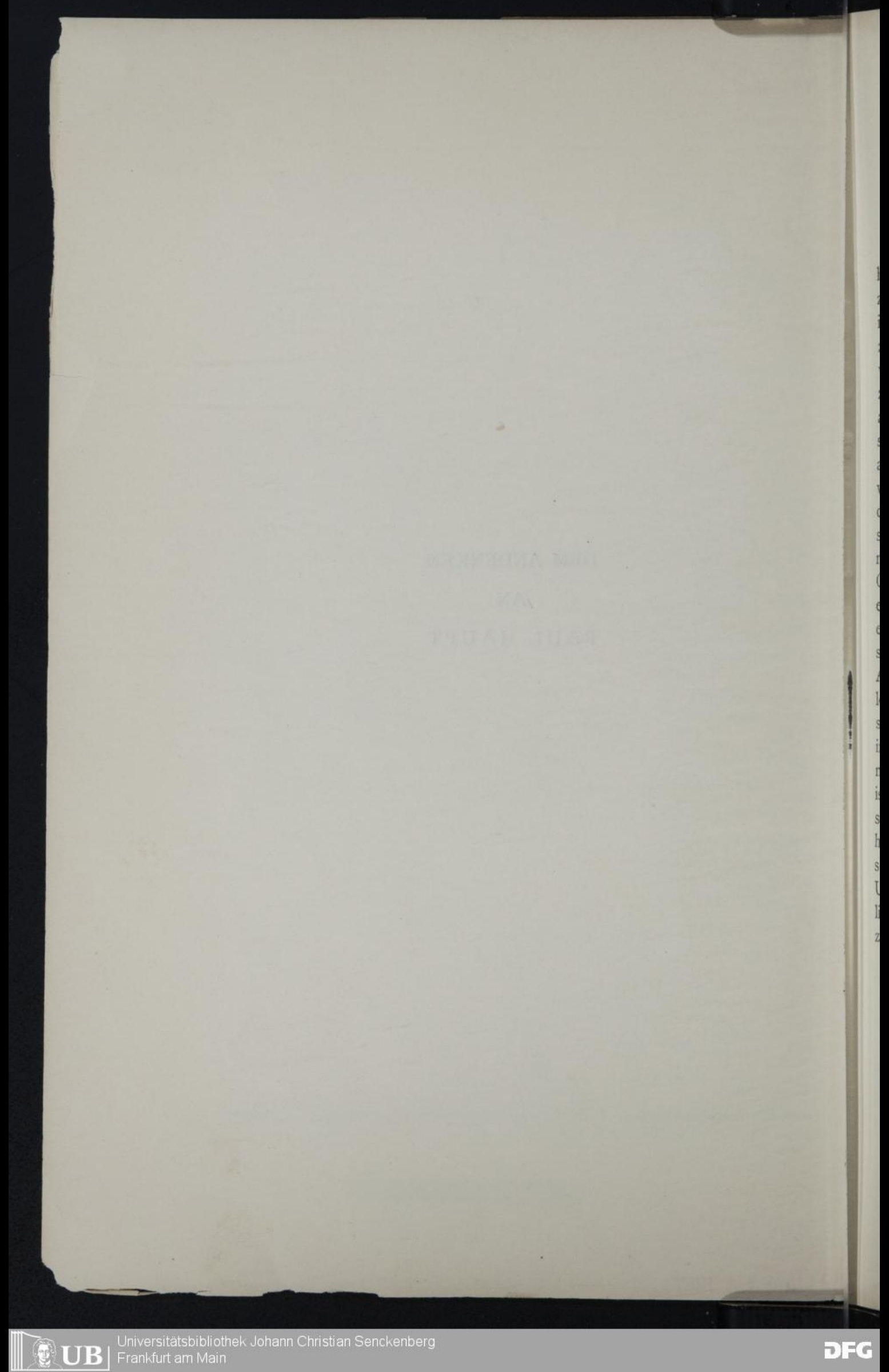


\section{VORWORT.}

In der folgenden "Chronologie der Könige von Israel und Juda“ habe ich den Versuch gemacht, auf anderen als den meistens bevorzugten Wegen zu genauer Fixierung der Regierungszeiten aller israelitischen und jüdischen Könige von Salomo bis Hosea bezw. Ședekia zu gelangen. Ausgangspunkt meiner Arbeit war die, wie ich glaube, wohl begründete Überzeugung, daß die "Babylonische Chronik" dazu zwinge, die israelitisch-jüdischen Synchronismen des Alten Testaments anders zu bewerten als gemeinhin geschieht. Im Verlaufe der Untersuchung gewann ich dann die Erkenntnis, daß Josephus oftmals Varianten oder Erweiterungen bietet, deren Beachtung bisher für unüberwindlich geltende Schwierigkeiten beseitigen hilft. Dies beides, meine durch die Anlage analoger keilschriftlicher Quellen bestimmte Einstellung zu den Zeitangaben des biblischen Königsbuches und eine zunehmende Schätzung der Archäologie des Josephus, war daher für den Gang der Untersuchung maßgebend und ließ in meinen Ausführungen einige schon häufiger erörterte und mir im Augenblicke weniger wichtig erscheinende Probleme der alttestamentlichen Chronologie bezw. der Geschichte Israels und Judas mehrfach in den Hintergrund treten. Meine Arbeit weist somit eine ungewöhnliche Gliederung auf und läuft vielleicht Gefahr, einseitig genannt zu werden. Wenn ich sie dennoch schon jetzt und fast unverändert in der Gestalt veröffentliche, die sie in den wenigen Wochen ihrer Entstehung gleichsam von selbst annahm, so möge das Wort bis dat qui cito dat gelten: der Assyriologe ist ja heutzutage allein mit der Durcharbeitung des sich alljährlich stark vermehrenden Materials an neuen Texten und der Verfolgung der hierbei ständig neu auftauchenden Probleme der verschiedensten Art so sehr überlastet, daß er kaum je voraussehen kann, wann und ob er Untersuchungen wieder aufnehmen und abrunden kann, die wie die vorliegende nur ein Grenzgebiet seines eigentlichen Forschungsbereiches zum Gegenstand haben.

Gießen, im Juli 1927.

JULIUS LEWY. 


\section{Abkürzungen :}

$\mathrm{AOTU}=$ Altorientalische Texte und Untersuchungen, herausgegeben von Bruno Meißner. Leiden $1916 \mathrm{f}$.

FAGV = Lewy, Forschungen zur alten Geschichte Vorderasiens. Leipzig I925 (MVAG 1924,2).

KAV $=$ Schroeder, Keilschrifttexte aus Assur verschiedenen Inhalts. Leipzig 1920. MVAG $=$ Mitteilungen der Vorderasiatischen Gesellschaft.

ZA N.F. = Zeitschrift für Assyriologie. Neue Folge.

ZDMG = Zeitschrift der Deutschen Morgenländischen Gesellschaft. 
Wenn die führenden Vertreter der alttestamentlichen Wissenschaft bei ihren Versuchen zur Rekonstruktion der Chronologie Israels und Judas die israelitisch-jüdischen Synchronismen der sogenannten Einleitungsformeln des Königsbuches erst in letzter Linie heranzuziehen pflegen, weil sie diese Synchronismen a priori für sekundär, für das Ergebnis künstlicher Rechnung halten, so kann der Assyriologe dem nicht beistimmen. Er muß vielmehr gerade umgekehrt vermuten, daß bereits sowohl das „Buch der Geschichte der Könige von Israel“ als auch die entsprechende Chronik der jüdischen Könige von Anfang an synchronistischen Charakter hatten.

Anstatt in eine theoretische Polemik gegen die herrschende Auffassung einzutreten gebe ich an dieser Stelle sogleich zwei ziemlich beliebig herausgegriffene Abschnitte der sogenannten „Babylonischen Chronik" wieder, aus denen sehr schnell erhellen dürfte, wie altorientalische Chroniken aussahen bezw. wie ihre Synchronismen zu bewerten sind:

Kol. I, I ff.

„[Im Jahre 3 des Nabonassar,] Königs von Babel, setzte sich Tiglatpileser in Assyrien auf den Thron. Im selben Jahre zog der [König von Assyrien] nach Akkad hinab und plünderte die Stadt Rabbilu und die Stadt Hamranu aus; auch führte er die Götter von Šapazza fort.

Im Jahre 5 des Nabonassar setzte sich Ummanigaš in Elam auf den Thron.

Im Jahre 14 des Nabonassar wurde er krank und verschied in seinem Palaste. I4 Jahre regierte Nabonassar über Babel. Nadin, sein Sohn, setzte sich in Babel auf den Thron.

Im Jahre 2 des Nadin wurde er in einem Aufstande getötet. Zwei Jahre regierte Nadin über Babel. Der Statthalter Šum-ukīn, der Urheber des Aufstandes, setzte sich auf den Thron. Einen Monat zwei Tage (?) regierte Śum-ukīn über Babel. Ukīn-zēr [....] stieß ihn vom Thron und usurpierte den Thron.

Im Jahre 3 des Ukīn-zēr verwüstete Tiglatpileser, indem er nach Akkad hinabzog, Bït-Amukanu, auch erreichte er den Ukīn-zēr. Drei Jahre regierte Ukīn-zēr über Babel. Tiglatpileser setzte sich in Babel auf den Thron. 
Im Jahre 2 des Tiglatpileser verschied er im Țebet. $\left(x^{x}\right)$ Jahre regierte Tiglatpileser über Akkad und Assyrien. Z $Z_{\text {wei }}$ Jahre (davon) regierte er in Akkad (selbst). Am 25. Tebet setzte sich Salmanassar in Assyrien [und Akkad(?)] auf den Thron. Šm(?)arain verwuistete er."

Kol. III, $19 \mathrm{ff}$.

„Im Jahre 4 des Mušēzib-Marduk am I 5 . Nisan wurde Menanu, König von Elam, vom Schlag getroffen. Sein Mund wurde betroffen und so konnte er nicht (mehr) sprechen. Am I. Kislew wurde die $\mathrm{Stadt}^{2}$ genommen, Mušēzib-Marduk wurde gefangen und nach Assur fortgeführt. 4 Jahre regierte Mušezzib-Marduk über Babel. Am 7. Adar verschied Menanu, König von Elam. 4 Jahre regierte Menanu über Elam. Hummahaldašu setzte sich in Elam auf den Thron."

Die „synchronistische“ Arbeitsweise des Babyloniers liegt auf der Hand: über die Babylonien unmittelbar betreffenden Ereignisse hinaus nimmt er von jedem Regierungswechsel nicht nur im benachbarten Assyrien, sondern sogar auch in dem damals nicht ganz so bedeutenden Elam in der Weise Notiz, daß er jede Thronbesteigung und jedes Regierungsende an der Stelle bucht, die der natürliche Ablauf der Dinge jeweils vorschreibt. Darüber hinaus wird ihm der Eintrag über das Ende eines Königs zum Anlaß, nach bestem Können zu berechnen und zu vermerken, wieviel Jahre die Regierung des betreffenden Herrschers gedauert hat. Mit anderen Worten: das Primäre sind die Synchronismen, sekundär und Rechenfehlern, Abrundungen ${ }^{3}$ und dgl. leichter unterworfen sind die Angaben über die absolute Regierungsdauer.

Wenn also Rühl zu Beginn seiner noch heute als grundlegend geltenden "Chronologie der Könige von Israel und Juda“4 gesagt hat, „weder hatten die Könige von Israel Veranlassung, notieren zu lassen, welche Könige von Juda zu ihrer Zeit regierten, noch die von Juda, wer ihre Kollegen in Israel waren und wann diese wechselten", so wird man dieses Verdikt gegen "den Synchronisten" so lange als unbegründet betrachten müssen, bis der Nachweis erbracht ist, daß die israelitischen und jüdischen Annalisten anders arbeiteten als die babylonischen. Dieser Nachweis, der übrigens schon Rühl oblag, da die „Babylonische Chro-

I Anstatt die Zahl der Regierungsjahre Tiglatpilesers anzugeben hat der Schreiber hier eine Lücke gelassen, vgl. Delitzsch, Abh. Sächs. Ges. d. Wiss, Bd. 25 (I906) phil.hist. Kl. Nr. I S. $19^{2} ; 25^{1}$.

2 D. i. Babel.

$3 \mathrm{Vgl}$. Delitzsch a. a. O. S. $19^{\mathrm{x}} ; 22^{\mathrm{x}} ; 224 ; 23^{\mathrm{x}}$.

4 Deutsche Zeitschrift f. die Geschichtswissenschaft Bd. I2 (I894) S. 44 ff.; I7I.

Vgl. Kittel, Die Bücher der Könige (I900) S. XI. 
nik" bereits 1890 allgemein zugänglich wurde, ${ }^{x}$ wird freilich schwer zu erbringen sein: wie sonst, z. B. in den formelhaften Wendungen privater oder amtlicher Briefe, ${ }^{2}$ besteht ja auch hier zwischen alttestamentlicher und babylonischer Ausdrucksweise eine so weitgehende Ähnlichkeit, daß direkter oder indirekter babylonischer Einfluß auf Israel angenommen werden muß. Auch kann kaum geltend gemacht werden, daß die synchronistische Annalistik, wie sie uns in der "Babylonischen Chronik“ entgegentritt, zu jung sei, als daß sie für das Vorbild der Quellen des alttestamentlichen Königsbuches angesprochen werden dürfte. Denn „die Tafelserie "Babylonische Chroniks" 3 bringt trotz aller sonstigen Ungleichmäßigkeit ihrer einzelnen Teile auch bereits in den Abschnitten, die im Unterschied von dem oben angezogenen Bericht über die Jahre 745 bis 668 v. Chr. das 27. bis 21 . und das 12. bis 9. und die erste Hälfte des 8. Jahrhunderts behandeln, ganz präzise Jahresangaben (z. B. „im II. Jahre [scil. des Sargon, d. i. ca. 264I] “ oder „im Jahre 5 des Eulmaššakin-šumi [ca. IOI I]"), und die Synchronismen reichen gleichfalls weit zurück, wenngleich sie in den dem Ausgang des 3. Jahrtausends gewidmeten Teilen nur so allgemein lauten wie „Ilušumma, König von Assur, (hat) zur Zeit des Suabu..... Schlieblich haben die Grabungen in Assur auch eine bis ins I2. Jahrhundert hinaufführende synchronistische Königsliste geliefert, die das gegenseitige zeitliche Verhältnis der einzelnen Herrscher zweier benachbarter Länder genau zu kennzeichnen vermochte, obwohl sie auf die Angabe der jeweiligen absoluten Regierungsdauer verzichtete. 4

Scheint es somit im Hinblick auf das, was der außerisraelitische Alte Orient lehrt, erlaubt, die israelitisch-jüdischen Synchronismen sowohl grundsätzlich für vertrauenswürdig als auch für ebenso ursprünglich, wenn nicht sogar für noch ursprünglicher zu halten, als die sie begleitenden, möglicherweise nachträglich hinzugefügten oder abgerundeten Angaben über die Regierungsdauer der einzelnen Herrscher, so muß füglich einmal versucht werden, die ältere Chronologie Israels und Judas in erster Reihe auf sie und die sie unmittelbar ergänzenden assyrisch-israelitischen Synchronismen der Keilschriftquellen zu gründen.

I In Schraders „Keilinschriftlicher Bibliothek“ Bd. II S. 274 ff. Spätere Übersetzung insbesondere bei Delitzsch a. a. O. Die neueste Übersetzung von Ebeling in Greßmanns „Altorientalischen Texten und Bildern zum A.T. 2 " I S. 359 f. ist nicht ohne Versehen und zudem gekürzt, ohne daß der Leser hierauf aufmerksam gemacht würde.

2 Für Schreiben privaten Charakters s. FAGV p. 50 I, mit den Briefadressen Ezra 4, I7 und des Elephantine-Papyrus Nr. I vgl. z. B. die Eingangsformel ana Šamašhașir, Sinmušallim u tappēšunu umma Hammurapima bei Thureau-Dangin, Revue d'Assyriologie Bd. 21 (1924) S. $24 \mathrm{ff}$.

$3 \mathrm{Zu}$ dieser vgl. jetzt Landsberger und Bauer ZA N. F. 3 (37) S. $6 \mathrm{I}-66$.

4 Vgl. hierzu unten S. 29. 
Wenn die sonst bevorzugten Angaben über die absolute Regierungsdauer der einzelnen Könige hierbei naturgemäß auch nicht ganz entbehrt werden können, so wird folgerichtig ihre Benutzung immerhin in der Weise einzuschränken sein, daß sie nur dort zur Ergänzung bezw. zur Nachprüfung der Synchronismen herangezogen werden, wo sie voraussichtlich zuverläßlich sind, d. h. insbesondere dort, wo Usurpationen, Doppelregierungen oder sonstige Ereignisse, die die antiken Rechnungen nach Königsjahren erfahrungsgemäß ungünstig beeinflussen, unseres Wissens nicht oder nur in geringem Umfange stattgefunden haben. Im übrigen muゆ selbstverständlich auch bei dem hier vorgeschlagenen Verfahren von Anfang an beachtet werden, daß die $146 \% / 12$ Jahre, die die Addierung der für die Zeit von Jehu bis zum Falle Samarias für Israel überlieferten Einzelposten bekanntlich ergibt, ${ }^{x}$ auf Grund der keilschriftlichen Nachrichten auf höchstens 120 Jahre verringert werden müssen, d. h. auch wo wir die überlieferten Regierungszahlen annehmen werden, werden wir in ihnen zunächst nach oben obgerundete Maximalangaben vermuten müssen, und wo die Überlieferung der absoluten Regierungszahlen Varianten aufweist, werden wir die niedrigere Jahressumme bevorzugen, und zwar ganz besonders falls diese mit der aus den Synchronismen zu errechnenden übereinstimmt.

\section{II}

Gehen wir im Hinblick auf den bekannten Bericht des Salmanassar über seinen vierten Feldzug gegen Damaskus $\left(84 \mathrm{I}\right.$ v. $\mathrm{Chr}^{2}$ ) bezw. über Jehus Huldigungsgeschenk - zunächst hypothetisch - davon aus, daß Jehus I. Regierungsjahr das Jahr $84 \mathrm{I} / \mathrm{O}$ v. Chr. ist, so ergibt sich bei Beachtung des letzten der soeben entwickelten Grundsätze für einen ersten Abschnitt der Epoche zwischen 84I und 722 folgende Liste:

Israel Juda

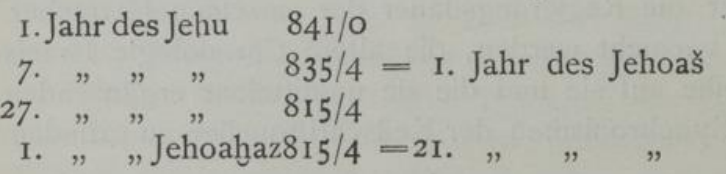

s. II Reg II,4; 12,2

s. Josephus Arch. IX $\$ 160 ; \$ 173^{3}$

I S. Kittel, Geschichte des Volkes Israel6 Bd. 2 S. 213; Sellin, Geschichte des israelitisch-jüdischen Volkes, Teil I S. 264.

2 So mit Forrer, MVAG 1915, 3 S. 27, Ungnad, Babylonisch-assyrische Grammatik 2 S. 143, Meißner, Könige Babyloniens und Assyriens S. I4I und anderen. Der neue assyriologische Bearbeiter der zweiten Auflage von Grebmann-Ungnad-Rankes „Altorientalischen Texten und Bildern zum A.T.", der auch sonst der neueren assyriologischen Literatur viel zu wenig Beachtung geschenkt hat, datiert Salmanassars Berichte zu unrecht noch in der früher üblichen Weise.

3 Anstatt dieses Synchronismus, der mit der im S 160 vorangegangenen Angabe über 
Israel

Juda

I7. Jahr d. Jehoahaz 799/8

$\{$ s. IIReg I3, IO; Josephus

des Jehoaš a. a. O. \$ 177

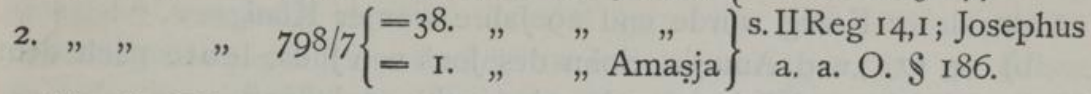

Für die Zeit des Jehu und seiner beiden Nachfolger auf der einen, des Jehoaš von Juda auf der anderen Seite kannte also noch Josephus eine in sich folgerichtige "synchronistische Überlieferung“, die Jehoaš von Juda 38 Regierungsjahre zuspricht. Das Verhältnis dieser Überlieferung zu derjenigen des Masoretischen Textes ist dabei, wie man leicht sieht, ein ganz charakteristisches: in ihrem zweiten Teil, d. h. in den beiden Synchronismen von II Reg I3, IO und I4,I, erscheint sie vollkommen unverändert auch im Masoretischen Texte, aber in ihrem ersten Teil ist sie hier (II Reg 12,2 und I3,I) in der Weise abgeändert, $\mathrm{da} ß$ die zu erwartende absolute Zahl der Regierungsjahre des Jehoaš um 2 Jahre vermehrt und die Zahl „2 I. Jahr" des dieser absoluten Zahl folgenden Synchronismus folgerichtig ebenfalls um 2 Jahre auf "23. Jahr" erhöht ist. Dieses eigenartige Verfahren, eine absolute Regierungszahl zu erhöhen und einen benachbarten Synchronismus - und zwar nur einen einzigen, aber nicht auch die weiterhin folgenden! an der Erhöhung teilnehmen zu lassen, wird uns, wie gleich hier bemerkt sei, noch öfter begegnen. ${ }^{x}$

III

Die Chronologie der jüdischen Könige Amașja und Azarja, denen wir uns nunmehr zuwenden müssen, bereitet bekanntlich besondere Schwierigkeiten. Wenn diese mit einem $\mathrm{m}$. E. verhältnismäbig sicheren Ergebnis behandelt werden können, so verdanken wir das den eigenartigen Notizen, mit denen II Reg I4,I7 und II Reg I4,2 I f. die offenbar der Interpretation bedürftige Zahlenangabe der "Einleitungsformel" zur Geschichte Amașjas wenigstens indirekt aufklären, sowie den nicht minder wertvollen Angaben, durch die Josephus den Bericht des Königsbuches noch weiter ergänzt bezw. die "Einleitungsformel" zu Azarja-Uzzija (II Reg $15, \mathrm{I}$ ) variiert hat.

die Dauer der Regierung Jehus harmoniert, bietet II Reg 13,I bekanntlich die Gleichung "I. Jahr des Jehoahaz $=23$. Jahr des Jehoaš", ein Synchronismus, der nicht einmal zu der nach den oben dargelegten Grundsätzen a priori weniger wahrscheinlichen Angabe von II Reg I0,36 stimmt, der zufolge Jehu 28 Jahre zukämen. Vgl. hierzu sofort.

I Die Ursache der Aufrundung der 38 Regierungsjahre des Jehoaš auf 40 ist möglicherweise nur in der bekannten Vorliebe für die Zahl 40 zu suchen, die dem alten und dem modernen Orient gemeinsam ist und die auch die Meša'-Inschrift (Z. 8) für chronologische Zwecke unbrauchbar macht. Für das Analogon der dem Jerobeam II. zugeschriebenen 40 (!) Jahre (statt 37 ) s. unten S. I $9^{1}$. 
Die Angaben des Masoretischen Textes lauten:

a) I4, I f.: „im Jahre 2 des Joaš, Sohnes des Jehoahaz Königs von Israel, wurde Amașjahu, Sohn des Joaš Königs von Juda, König. ${ }^{2} 25$ Jahre war er alt, als er König wurde und 29 Jahre war er König. . . “

b) I4, I7: „und Amașja, Sohn des Joaš von Juda, lebte nach dem Tode des Jehoaš, Königs von Israel, (noch) 15 Jahre.“

c) I4,2If.: "und das ganze Volk von Juda nahm den Azarja, während derselbe (erst) I 6 Jahre alt war, und machte ihn zum König an Stelle seines Vaters Amașjahu. ${ }^{22}$ Derselbe hat Elat befestigt und brachte es an Juda zurück, nachdem sich der König zu seinen Vätern gelegt hatte."

d) I 5, I : „im Jahre 27 des Jerobeam, Königs von Israel, wurde Azarja, Sohn des Amașja Königs von Juda, König.“

Aus der hier unter d) angeführten Angabe könnte man schließen, daß Amașja erheblich länger als 29 Jahre (s. sub a) regiert haben müsse, weil außer den hier genannten 27 Jahren Jerobeams nach II Reg 13, $10+14, \mathrm{I}$ ja auch etwa 14 Jahre des Jehoas von Israel in seine Zeit fallen sollten. Diese in der Tat häufig angenommene Folgerung wäre indessen voreilig; denn Josephus (IX $\$ 216)$ gibt den nach II Reg 14,2 zu erwartenden Synchronismus

I. Jahr des Azarja = I4. Jahr des Jerobeam.

Somit ist es ratsam, I5,I als unsicher überliefertes bezw. schon im Altertum angezweifeltes Zeugnis erst später zu verwerten.

I4,22 (s. oben sub c) läßt den Verdacht entstehen, daß Azarja zu Lebzeiten seines Vaters die Regierung übernommen habe; denn wer bei der Notiz über die Befestigung von Elat ausdrücklich bemerkte, daß dies Ereignis „nach dem Tode des Königs“ eintrat, scheint auch Taten gekannt zu haben, die Azarja vor dem Tode Amașjas vollführte.

$\mathrm{Zu}$ einem ganz ähnlichen Schluß führt denn auch 14,2I (s. oben unter c): im Unterschied von I5, I zeigt ja auch dieser Passus wenigstens mittelbar, daß die Thronbesteigung Azarjas unter ungewöhnlichen Umständen vor sich ging. Vergleicht man nämlich den ganz ähnlichen Bericht über die Thronbesteigung des Šallum (Joahaz) im Jahre 609 (II Reg 23,30 $\mathrm{O}^{\mathrm{bx}}$ : ,und das Volk des Landes nahm den Jehoahaz, einen Sohn des Josia, <und salbte ihn> und machte ihn zum König an Stelle seines Vaters"), so darf man - speziell im Hinblick auf die in beiden Fällen gleichermaßen hervorgehobene Mitwirkung des 'am-hā'āreș vermuten, daß Azarja seinen Vater ersetzte, als sich Juda in einer Krise befand, die der durch die Schlacht von Megiddo bezw. durch Josias Tod gekennzeichneten Katastrophe des Jahres 609 irgendwie ähnlich gewesen ist.

I Vgl. II Chr. 36,I und dazu meine Ausführungen FAGV S. 2 I ff. 
Tatsächlich ist nun Juda unter Amașja in eine schwierige Lage geraten, die der des Jahres 609 durchaus vergleichbar ist, s. II Reg I4, I I ${ }^{\mathrm{b}} \mathrm{ff}$ : , und Jehoaš, König von Israel, zog heran, und er und König Amașjahu von Juda standen einander gegenüber bei Bēt-Šemeš, das zu Juda gehört, und Juda wurde von Israel geschlagen und sie flohen ein jeder in seine Heimat. Und den Amașjahu, König von Juda, Sohn des Jehoaš Sohnes des Ahazjahu, nahm Jehoaš, König von Israel, in BētŠemeš gefangen. Und er $\mathrm{kam}^{x}$ nach Jerusalem und riß an der Mauer von Jerusalem - vom Ephraimtore bis zum Ecktore - 400 Ellen nieder. Und er nahm alles Gold und Silber und alle Geräte, die sich im Tempel Jhwhs und in den Schatzkammern des königlichen Palastes befanden, und Geiseln und kehrte nach Samaria zurück."

Im übrigen wird auch noch von ganz anderer Seite her wahrscheinlich, daß Azarjas Thronbesteigung nicht in der zu normalen Zeiten üblichen Weise erfolgt ist: bekanntlich erscheint Azarja meist nicht unter diesem seinem ursprünglichen Namen, sondern als Uzzija; Doppelnamen bezw. die Annahme eines besonderen Thronnamens begegnen aber, wie innerhalb der Geschichte Babyloniens und Assyriens z. B. derFall des Ašsurețel-iläni-mukīn-apli-Assarhaddon, innerhalb der jüdischen Geschichte die Fälle des Jehojaqim-Eljaqim, Jehojachin-Jechonja, Mattanja-Șidqijahu und wiederum des Jehoahaz-Šllum lehren, regelmäßig dann, wenn die regelmäßige Thronfolge in irgendeiner Weise unterbrochen wurde. ${ }^{2}$

Während der biblische Bericht II Reg I4, I I ${ }^{b} \mathrm{ff}$. leider nicht sagt, in welchem Jahre sei es des Amașja, sei es des Jehoaš Amașja gefangen und Jerusalem geplündert wurde, erklärt Josephus (IX $\$ 203$ ) sehr bestimmt: „dies geschah mit den Jerusalemern im I4. Regierungsjahre des Amașja". Da dieser Angabe a priori zu mißtrauen kein Grund vorliegt, eröffnet sich von hier aus eine erste Möglichkeit zur Ergänzung der oben unter II aufgestellten Liste und wir erhalten folgende Daten:

$$
\text { Israel Juda }
$$

\section{Jahr des Jehoaš $799 / 8$}

2. " " " $\quad 798 / 7=$ I. Jahr des Amașja

I5. " " " $\quad 785 / 4=$ I4. " " " Niederlage des Amașja

I6. , " $, \quad 784 / 3 \quad, \quad " \quad$ letztes Jahr des Jehoas [s. II Reg ${ }_{13}, \mathrm{IO}^{\mathrm{b}}$
I.
, Jerobeam $784 / 3=\mathrm{I} 5$.
s. II Reg 14,23.

$x \mathrm{Da}$ das Qere mit II Chr 25,23 und LXX zu verwerfen sei, ist mir nicht wahrscheinlich. Denn im Vordergrund der Erzählung steht natürlich Jehoaš' Marsch nach Jerusalem, nicht der verhältnismäßig nebensächliche Umstand, daß er Amașja als Gefangenen mit sich führte. (Anders freilich Josephus [ $\$ 200 \mathrm{ff}$.], dessen Bericht jedoch nach später Ausmalung aussieht.)

2 Für das Jehojaqim und seine Nachfolger betreffende Material s. FAGV p. 2 I f.; $48 \mathrm{ff}$; $58 \mathrm{f}$.; $58 \mathrm{f}$; 63 , für das Assarhaddon betreffende Streck, Assurbanipal Bd. I (I916 S. 223 f.; Schmidtke, AOTU I, 2 (I916) S. 86 ff. 
Jehoaš ist also sehr bald nach seinem Siege über Juda gestorben, und mit II Reg 14,2 (s. oben unter a) verglichen, ist somit auch die eigenartige Angabe II 14,I7 (s. oben unter b), der zufolge Amașja den Jehoaš um 15 Jahre überlebt haben soll, chronologisch einwandfrei; insofern man hier statt „I5 Jahre“ lieber „I4 Jahre“ lesen würde, liegt die Ungenauigkeit ja innerhalb der Fehlergrenze von I Jahr, die die übliche Abrundung jedes Anfangs- und jedes Endjahres auf volle Jahre auch hier eo ipso mit sich bringt. Damit erlangt 14,17 aber auch den Wert eines dritten mittelbaren Zeugnisses für eine nur I4 jährige Regierung des Amașja: die Notiz besagt ja nicht etwa, daß Amașja nach Jehoaš Tode noch I5 Jahre „regierte“, sondern nur, daß er I5 Jahre länger als jener gelebt hat.

IV

Mag man die im vorigen Abschnitte gegebene Interpretation von II Reg I4, I7 und I4,2 I f. in jedem Punkte für unanfechtbar halten oder nicht - Tatsache ist, daß der oben abgeleitete neue Synchronismus letztes Jahr des Amașja

$=$ erstes Jahr des Azarja $\}=785 / 4=15$. Jahr des Jehoaš von Israel

nicht nur ungezwungen erklärt, warum Azarja bei seiner Erhebung zum König erst 16 Jahre alt war ${ }^{x}$, sondern auch die schwierige Chronologie der letzten israelitischen Könige zu einem erheblichen Teile aufzuhellen geeignet ist. Denn da die II Reg 15 vorliegende Überlieferung die Antrittsjahre der meisten dieser Herrscher mit Hilfe der Ära Azarjas fixiert hat, ergibt sich nunmehr unmittelbar folgende, durch die assyrischisraelitischen Synchronismen überraschend gut erhärtete Fortsetzung der oben unter II berechneten Liste:

$$
\text { Juda Israel }
$$

I. Jahr des Azarja 785/4 = 15. Jahr des Jehoaš

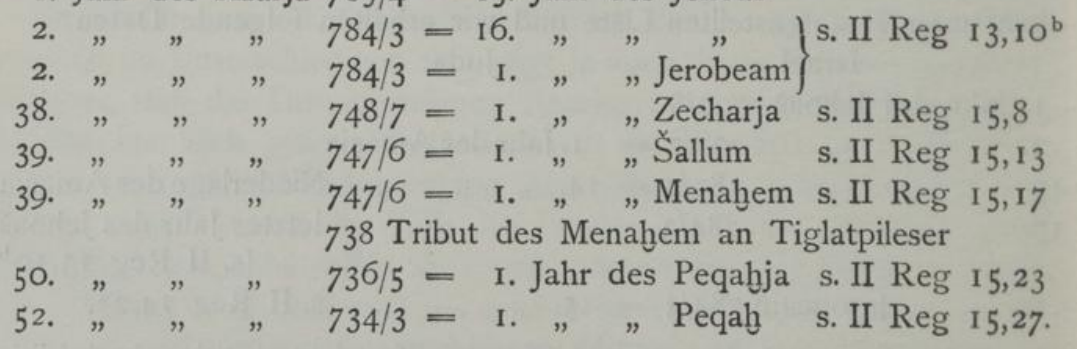

\section{V}

I. Eine unmittelbare Ergänzung erfährt die obige Liste durch die bekannte assyrische Angabe (Tigl. Pil. Kleine Inschr. I 7 f.) über Peqahs Ablösung durch Hosea, d. h. wir erhalten

× S. II Reg 14,21. 
52. Jahr des Azarja 734/3 = I. Jahr des Peqah (II Reg 15,27)

$$
733 / 2=\text { I. " , Hosea. }
$$

Hierzu bietet nun II Reg I7, I den Synchronismus

„12. Jahr des Ahaz = I. Jahr des Hosea [733/2]“.

Prüfen wir diese letzte Gleichung nach, so ergibt sich jedoch, daß wir vielmehr

$$
\text { "9. Fahr des Ahaz = I. Fahr des Hosea [733/2]" }
$$

erwarten müßten; denn die in ihren letzten Abschnitten durch die neubabylonisch-jüdischen Synchronismen voll gesicherte und auch sonst unverdächtige (postdatierende) Liste der letzten jüdischen Könige hinauf

\begin{tabular}{|c|c|c|c|c|c|c|}
\hline I. & $"$ & $"$ & , & Jechonja & $597 / 6$ & s. die Nachweise in \\
\hline I. & " & $"$ & " & Jojaqim & $608 / 7$ & FAGV p. $23 \mathrm{ff}$. \\
\hline I. & " & ” & ” & Josia & $639 / 8$ & \\
\hline 2. & $"$ & $"$ & ” & Amon & $640 / 39$ & s. II Reg 2I IO \\
\hline I. & $"$ & $"$ & $"$ & $"$ & $641 / 0$ & S. 11 Keg 21,19 \\
\hline 55. & $"$ & " & " & Manasse & $642 / I$ & s. II $\operatorname{Reg} 2 \mathrm{I}, \mathrm{I}$ \\
\hline I. & $"$ & " & " & & $696 / 5$ & S. $110 \mathrm{~kg} 21,1$ \\
\hline 29. & " & ", & , & Hizkia & $697 / 6$ & s. II $\operatorname{Reg} 18,2$ \\
\hline I. & " & $"$ & $"$ & $"$ & $725 / 4$ & \\
\hline I6. & " & $"$ & . & Ahaz & $726 / 5$ & s. II $\operatorname{Reg} 16,2$ \\
\hline 12. & $"$ & " & ", & $"$ & $730 / 29$ & \\
\hline 9. & " & $"$ & $"$ & " & 12 & \\
\hline
\end{tabular}
bis zu Ahaz enthält folgende Zahlen:

Der Synchronismus Ahaz-Hosea, den die „Jüdische Königsliste“ fordert, weicht also von demjenigen, den II Reg I7,I tatsächlich bietet, um 3 Jahre ab, d. i. die gleiche Anzahl von Jahren, um die der Verf. von II Reg 1 7,6 irrt, wenn er behauptet, das Jahr der Einnahme von Samaria - nach den assyrischen Quellen bekanntlich zweifellos das Jahr 722/I sei das 9. Jahr Hoseas. Mit anderen Worten: untereinander übereinstimmend korrigieren II Reg $17, \mathrm{I}$ und 6 die sowohl von den anderweitigen jüdischen als auch insbesondere von den assyrischen Quellen gelieferte Chronologie Hoseas im Sinne einer (ja auch in neuerer Zeit mehrfach vertretenen) Auffassung, der zufolge Hoseas Regierung bis zur Einnahme Samarias gedauert haben soll. Und zwar hat die Korrektur nicht die offenbar furr unantastbar gehaltene Zahl von 9 Regierungsjahren Hoseas betroffen - die Zahl, an der als Minimum der Regierung Hoseas auch wir Heutigen auf Grund der assyrischen Nachrichten festhalten müssen -, sondern nur die durch die jüdische Königsliste an die Hand gegebenen beiden Synchronismen

$$
\begin{aligned}
& \text { 9. Fahr des Ahaz }[733 / 2]=1 \text {. (Antritts-) Fahr des Hosea und } \\
& \text { 18. " " " Hizkia }\}[724 / 3]=9 \text {. volles (letztes) Fahr des Hosea. }
\end{aligned}
$$


2. Wer die Zeit der israelitischen Könige statt im Jahre 724/3 erst mit dem Falle Samarias 722/I enden ließ, gleichwohl aber dem spätestens $733 / 2$ zur Herrschaft gekommenen Hosea nur 9 Jahre zusprach, dürfte folgerichtig die Regierungsdauer eines der Vorgänger des Hosea um die 3 Jahre verlängert haben, die er der Gesamtheit der Könige von Israel zulegen zu sollen glaubte. Prüfen wir demgemäß nunmehr, ob die oben S. 14 aus den Synchronismen mit Azarja von Juda abgeleitete Chronologie der Vorgänger Hoseas mit den Zahlen vereinbar ist, die II Reg I5,8 ff. als jeweilige absolute Regierungsdauer der gleichen Herrscher überliefert sind, so ergibt sich zunächst, daß eine den korrigierten Zahlen von II Reg I7,I und 6 konforme Änderung überhaupt nur bei der für Hoseas letzten Vorgänger überlieferten Zahl von angeblich 20 Regierungsjahren in Frage kommen kann. Durch Ergänzung der obigen Liste der Synchronismen um die soeben behandelten Daten Hoseas erhalten wir nämlich folgende Tabelle:

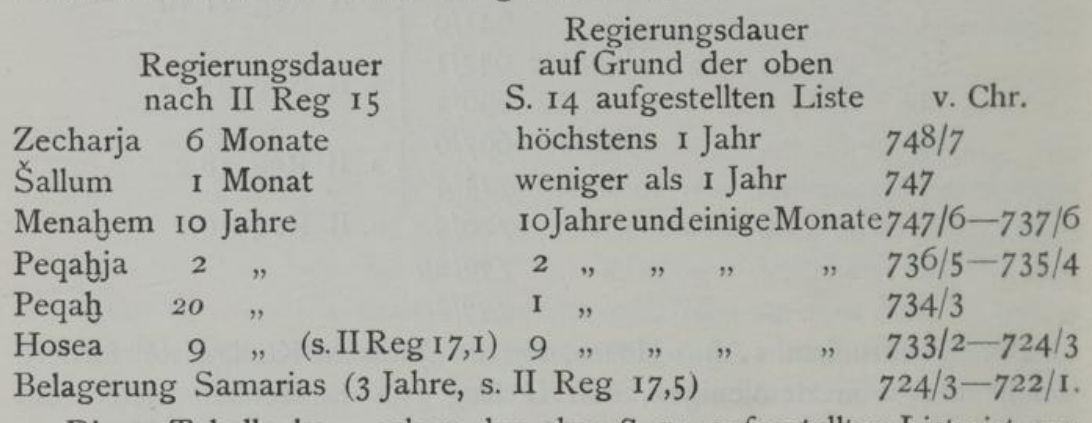

Dieser Tabelle bzw. schon der oben S. I4 aufgestellten Liste ist nun nicht nur zu entnehmen, daß die absoluten Regierungszahlen, die II Reg I $5,8 \mathrm{ff}$. für die israelitischen Könige überliefert sind, postdatierende Rechnung zur Voraussetzung haben wie die Quellen der oben S. 15 gegebenen Liste der jüdischen Könige, sondern auch daß, wie bereits erwähnt, allein bei Peqah b. Remalja Zweifel an der Ursprünglichkeit der überlieferten Zahl der Regierungsjahre erlaubt sind. Setzen wir daher den bereits entwickelten Gedankengängen gemä $h$, zu denen uns II Reg I 7,I und 6 Veranlassung gaben, des Peqah b. Remalja Regierungszeit zunächst hypothetisch und ohne Rücksicht auf weitere, die Vorgeschichte Peqahs berührende Folgerungen - statt auf 20 Jahre auf 17 Jahre an, so ergeben sich einerseits die doppelte Gleichung

17. (letztes) Jahr des Peqah $=734 / 3=52$. (letztes) Jahr des Azarja (s. oben S. 14), andererseits der Synchronismus

17. Jahr des Peqah $(=734 / 3)=$ I. Jahr des Abaz, s. II Reg I6, I.

Damit dürfte indessen bewiesen sein, daß der Urheber der unrichtigen Synchronismen von II Reg 17,1 und 6 wirklich, wie wir unter- 
stellten, die von ihm für notwendig gehaltene Hinabrückung Hoseas von $733 / 2-724 / 3$ auf $730 / 29-722 / 1$ dadurch auszugleichen versuchte, dal. er die ihm für Peqah b. Remalja überlieferte Regierungszeit um 3 Jahre verlängerte. Denn nunmehr schwebt ja der letzte Synchronismus der oben S. 14 wiedergegebenen Liste ,52. Jahr des Azarja $734 / 3=\ldots \ldots \ldots$. nicht mehr in der Luft; vielmehr ergibt sich, dal. das 52. und - nach II Reg 15,2 - gleichzeitig letzte Jahr Azarjas mit dem I. Jahr eines neuen jüdischen Herrschers in der zu erwartenden Weise zusammenfällt, und daß damit auch eine lückenlose Chronologie der israelitischen Könige von Jehu bis Hosea und der gleichzeitigen Könige von Juda gewonnen sein dürfte, die sowohl mit den assyrisch-israelitischen Synchronismen der Keilschriftquellen als auch mit den überlieferten israelitisch-jüdischen Synchronismen restlos harmoniert.

3. Neben der hiermit neu erhaltenen Gleichung

52. Jahr des Azarja 734/3 = I. Jahr des Ahaz

steht nun freilich noch die der "Jüdischen Königsliste“ (oben S. 15) zu entnehmende Gleichung

I. volles Regierungsjahr des Ahaz $=74 \mathrm{I} / \mathrm{O}$.

Dieses Nebeneinander bereitet jedoch deshalb keine unüberwindlichen Schwierigkeiten, weil wir aus II Reg 15,5 erfahren, daß Azarja aussätzig wurde und deshalb der Regierung entsagen multe: die jüdische Königsliste nennt uns mithin das Jahr, in welchem dem Ahaz als dem Sohne und Nachfolger des Jotam die Vertretung des seit 16 Jahren behinderten Azarja zufiel, während die synchronistische Überlieferung in II Reg I6,I die Regierung des Ahaz erst mit dem Ableben Azarjas beginnen lälbt. Beide Ưberlieferungen setzen damit übrigens nur das Verfahren fort, das sie schon II Reg I5, If. angewandt hatten; denn auch hier hat, wie aus den obigen Ausführungen über Amașjas Niederlage und die Thronbesteigung des Azarja ohne weiteres hervorgeht, die „Königsliste“, das ist die Überlieferung, die in II Reg I 5,2 zu Worte kommt, Azarjas Regierung mit seiner Erhebung durch den 'am-hā'āreș beginnen lassen, wogegen die ,synchronistische Überlieferung" ( $15, \mathrm{I}$ in der bei Josephus erhaltenen richtigen Fassung und I4,23) es wiederum vorgezogen hat, die Jahre Amașjas solange durchzuzählen, wie Amașja tatsächlich gelebt hat. ${ }^{x}$

4. Einen letzten und m. E. schlagenden Beweis für die Probehaltig-

I Wenn somit Regentschaftsjahre sowohl dem verhinderten eigentlichen Inhaber des Thrones als auch dem Regenten und erst designierten Könige zugerechnet werden, so ist das, wie man leicht erkennt, nur die konsequente Fortsetzung der für die ältere Überlieferung - ungefähr bis zur Mitte des 8. Jahrhunderts - so charakteristischen Methode der doppelten Zählung derjenigen Jahre, in denen ein Thronwechsel stattfand. Und wenn der Übergang zur postdatierenden Rechnung der Königsjahre, wie sie in Babylonien und Assyrien seit alters üblich war, zur Zeit des Azarja-Uzzija eintritt - in der "Jüdischen Königsliste" ist diese Zählungsweise, wie wir sahen, seit Jotam, in der „Israelitischen Lewy, Die Chronologie d. Könige v. Israel u. Juda. 
keit der obigen Aufstellungen dürften die nunmehr zu besprechenden Daten der Regierung Jotams liefern: die oben S. I5 angezogene postdatierende Liste der Könige von Juda ergibt als Jotams „Antrittsjahr“ (hebr. rēšīt malkūt oder haššanā hārīōōnīt = babyl. rēš sarrūti) das Jahr $75^{8} / 7$; denn sie bietet

$$
\begin{aligned}
& \left.\begin{array}{l}
\text { I6. volles Jahr des Ahaz 726/5 } \\
\text { I. " " " " } " 74 \mathrm{I} / \mathrm{O}
\end{array}\right\} \text { s. II Reg } 16,2 \\
& \text { I6. (letztes) ”, Jotam } 742 / 1 \text { \} } \\
& \text { I. volles " " " 757/6 }
\end{aligned}
$$

Der oben S. 14 abgeleiteten synchronistischen Liste zufolge sollte dieses Jahr 758/7 mit dem 27. Jahre Jerobeams II. zusammenfallen, da sich als dessen I. Jahr $784 / 3$ ergab. Und wirklich kennt II Reg I5, I einen Synchronismus zwischen dem 27. Jahr des Jerobeam und dem Antrittsjahr eines jüdischen Königs! Als Namen des in diesem Jahre zur Herrschaft gekommenen Dawididen nennt der überlieferte Text zwar nicht Jotam, sondern dessen Vater Azarja, aber wir sahen ja bereits (oben S. 12), daß man noch zu Josephus' Zeit diesen Synchronismus verwarf und vielmehr das I4. Jahr Jerobeams dem Antrittsjahre Azarjas gleichsetzte. Somit ist der Synchronismus des Masoretischen Textes (und bemerkenswerterweise auch der Versionen) nicht völlig falsch; die Unstimmigkeit besteht vielmehr nur in einem begreiflichen Abirren eines alten Abschreibers, der bei der Abschrift des für Azarjas Antritt geltenden Datums „I4. Jahr des Jerobeam" versehentlich die nächstfolgende, Jotams Regentschaftsantritt fixierende Angabe „27. Jahr des Jerobeam" einsetzte. ${ }^{x}$

Wenn schließlich der postdatierende letzte Teil der „Israelitischen Königsliste" Peqah b. Remalja 20 Jahre (ursprünglich vielmehr I7 Jahre, s. oben S. I6) zuweist, obwohl die assyrisch-israelitischen Synchronismen und, wie gleichfalls oben S. I4ff. gezeigt wurde, auch die Mehrzahl der jüdisch-israelitischen Synchronismen übereinstimmend dafür zeugen, daß ihm nur das eine offizielle Regierungsjahr 734/3 zuerkannt werden darf,

Königsliste" seit Zecharja gebräuchlich —, so ist das nicht weiter auffällig: gerade bei König Uzzija, der auch sonst ganz ungewöhnliche Tatkraft bewies und insbesondere auch nach assyrisch-babylonischem Vorbild persönlich zu opfern gedachte (s. den Bericht der Chronik II 26,2-2I), dürfen wir auch auf diesem Gebiete eine praktische Reform des Althergebrachten erwarten.

$x \mathrm{Ob}$ erst dieses Versehen dazu geführt hat, daß 15,32 den Synchronismus I. Jahr des Jotam = 2. Jahr des Peqah b. Remalja

bietet, wage ich nicht zu entscheiden. Jedenfalls darf dieser Synchronismus schon deshalb als unsicher bezw. als irrig bezeichnet werden, weil er 15,33 widerspricht, insofern er Jotam statt 16 voller Regierungsjahre nur 15 volle Regierungsjahre zuweist und insbesondere weil, wie wir sahen, die Regentschaft des Ahaz nicht erst im 17. Jahre des Peqah $=734 / 3$ [16,1], sondern schon im Jahre 741/0 (das wäre das 10. Jahr Peqahs) begonnen haben muß. Vollends spät und wohl erst aus $15,32+15,27$ (hierzu s. oben S. 15) abgeleitet ist schließlich die wertlose Gleichung II Reg 15,30 „Antrittsjahr des Hosea=20. Jahr des Jotam". 
so wird man daraus folgern dürfen, daß Peqah schon seit $750 / 49$ als Thronprätendent auftrat und wenigstens in einem Teile des Landes Anerkennung gefunden hatte. Zugunsten dieser Vermutung ist zunächst auf die Notiz II Reg I 5,37 zu verweisen, welche - ohne Peqah den Königstitel zuzugestehen! - behauptet, Peqah habe schon zur Zeit Jotams $[758-742]$ zu einer Gefahr für Juda zu werden begonnen. Und da Tiglatpileser (Kleine Inschr. I I 7 f.) Peqahs Ermordung durch Hosea sanktioniert hat, ist ja auch gewiß, daß Peqah als Exponent einer Assurfeindlichen bezw. Damaskus-freundlichen Politik auf den Thron gekommen ist, welche Menahems Huldigung vor Tiglatpileser widerrief. So spricht in der Tat nichts gegen die nächstliegende Annahme, dals Peqah sich schon gegen den Damaskus-feindlichen Jerobeam empört hat. ${ }^{x}$

Während hier freilich eine gewisse Unsicherheit bestehen bleibt, weil die Zusatzquelle II Reg 15,37 eben doch wenig ergiebig ist, dürfte eine anderweitige praktische Bestätigung des oben für Peqahs Usurpation abgeleiteten Datums nicht ohne jede Bedeutung sein, welche sich aus Jesaja zu ergeben scheint: Jesajas gegen den „Remalja-Sohn“ und seinen damaskenischen Bundesgenossen gerichteten Trostsprüchen an Ahaz geht bekanntlich die sogenannte Berufung des Propheten (Kap. 6) unmittelbar voraus, welche 6,1 in das "Todesjahr des Uzzija“ versetzt. Nach unseren obigen Aufstellungen ist dieses Jahr nun aber nicht nur das Jahr der Thronbesteigung Peqahs (was ja aus II Reg I5,27 seit jeher folgte), sondern gleichzeitig ein Jahr (das 8te) des Ahaz! Mit anderen Worten: die oben entwickelte Chronologie erklärt auch, warum Jesaja 6 und 7, zwei Kapitel, deren engste Zusammengehörigkeit seit langem für wahrscheinlich gilt, ${ }^{2}$ zwei, wie es bisher scheinen multe, verschiedene Datierungen erhalten konnten; sowohl die primitivere, aber dem Zeitgenossen ohne weiteres verständliche Zeitangabe „im Todesjahre des Uzzija“ als auch die II Reg 16,5 konformere „zur Zeit des Ahaz" besagen eben das Gleiche. ${ }^{3}$

I Jerobeam II. hat, wie die Synchronismen lehren (s. die Tabelle oben S. I4), von 784-748, also 37 Jahre regiert. Man darf vermuten, daß diese 37 Jahre - ähnlich wie die 38 Jahre des Jehoaš von Juda (s. dazu oben S. II $^{x}$ ) - sekundär zunächst zu der beliebten Zahl von 40 Jahren aufgerundet worden sind; denn Josephus gibt Jerobeam II. an Stelle der in jedem Falle unmöglichen absoluten Zahl von 4 I Jahren, die II Reg 14,23 hat, wiederholt (IX S 205 und 215) 40 Jahre. ${ }^{2} \mathrm{~S}$. Duhm, Das Buch Jesaia ${ }^{4}$ (I922) S. 64.

3 Obwohl die Stellen II Reg 18, r und 9 f. für die im obigen behandelte Chronologie der letzten Könige von Israel und ihrer jüdischen Zeitgenossen Azarja, Jotam und Ahaz bedeutungslos sind, sei doch wenigstens kurz darauf hingewiesen, da f ihre Angaben unter sich und gegenüber II Reg ${ }^{7} 7,1$ und 6 (s. dazu oben S. ${ }_{5}$ f.) folgerichtig sind und nicht durchaus wertlos zu sein brauchen; nichts hindert nämlich anzunehmen, daß I. Hizkia be. reits etwas länger als 2 Jahre Mitregent gewesen war, als er nach Ahaz' Tod im Verlaufe des Jahres 726/5 König wurde (s. die Tabelle oben S. 15), bezw. daß 2. in II Reg 18,I und 9 f. eine Zusatzquelle durchgedrungen ist, die die Regierung des Assyrien-feindlichen 
VI

Unterstellt man, daß die biblische Angabe über die absolute Regierungslänge des Jehoram b. Ahab (II Reg 3,I) richtig ist und daher den Ausgangspunkt zu liefern vermag, der für jede auf Synchronismen gegründete Chronologie unerläblich ist, so liefern die Synchronismen der Einleitungsformeln für die Zeit von Jehu an aufwärts bis zu dem durch die Spaltung des Reiches Salomos eo ipso gegebenen Synchronismus folgende Daten: Israel

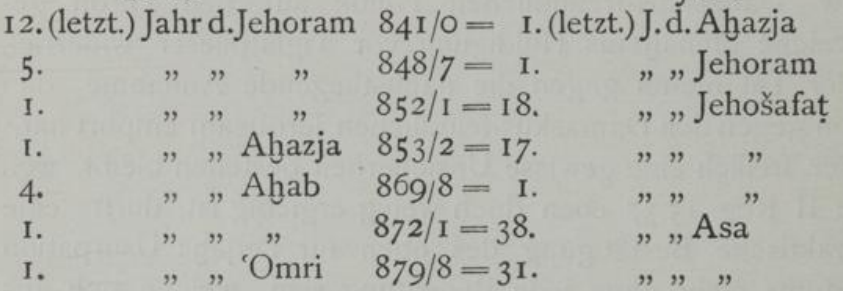

s.II $\operatorname{Reg} 8,25$

s. II Reg 8,16

s. II Reg 3,I

s. I Reg 22,52

s. I Reg 22,4 I

s. IReg 16,29

s. I Reg 16,23

frommen Hizkia vom Augenblicke seiner Erhebung zum Mitregenten des assyrisch gesinnten Ahaz an gerechnet und die Unternehmungen Salmanassars und Sargons gegen Samaria entsprechend unter dem 4. und 6. Jahre Hizkias notiert hatte, und daß 3 . wie II Reg 17,1 und 6 auch hier Hoseas Regierung irrig von $733 / 2-724 / 3$ auf $730 / 29-722 / 1$ heruntergerückt worden ist. Wir dürfen also die Synchronismen II Reg 18,1 und 9 f. teils als korrigiert, teils als sekundär bezw. interpoliert ausscheiden, erhalten aber im übrigen durch die Kombination von II Reg I8,I ud 9f. mit der "Jüdischen Königsliste“ (s. oben S. 15) die folgende Chronologie Hizkias:

$722 / 1=6$. volles Jahr des Hizkia als Mitregenten $=4$. volles Jahr des Hizkia als Alleinherrscher $724 / 3=4$. " " " " " " $725 / 4=3 . \quad " \quad, \quad$ " " ,

$726 / 5=2 . \quad " \quad " \quad, \quad$ = Antrittsjahr des Hizkia als Alleinherrscher $727 / 6=1 . \quad, \quad, \quad, \quad, \quad, \quad=15$. volles Jahr des Ahaz $728 / 7=$ Antrittsjahr , " ",$\quad=14 . \quad$ " , " .

Daß die kurze Mitregentschaft des Hizkia, die auf diese Weise wahrscheinlich wird, in der II Reg 18 ,2 genannten absoluten Zahl von 29 Jahren Hizkias nicht berücksichtigt ist, ist nur folgerichtig; denn im Gegensatz zu der älteren, noch nicht postdatierenden Chronographie, welche Regentschaftsjahre konsequenterweise doppelt zählte (s. oben S. $17^{x}$ ), schließt die seit Azarja rezipierte postdatierende Rechnungsweise solche zweimalige Zählung natürlich ebensosehr aus wie die doppelte Zählung der Todes- bzw. Antrittsjahre.

Auf der anderen Seite muß allerdings bemerkt werden, daß der an sich geringe Wert der Synchronismen von II Reg 18, I und 9 f. auch noch durch das Schwanken der Überlieferung beeinträchtigt wird: $z u \quad 18,1$

"3. [richtiger 6!] Jahr des Hosea $=$ Antrittsjahr des Hizkia"

liefert Josephus (IX $\$ 260$ ) die Variante

und $z$ i 18,10

"4. Fahr des Hosea $=$ Antrittsjahr des Hizkia"

"Jahr der Eroberung Samarias $[722 / \mathrm{r}]=6$. Jahr des Hुizkia" gibt er (IX S 278) die zu seiner Lesung von I8, I nicht einmal stimmende Gleichung und gibt LXX sogar "Fahr der Eroberung Samarias $=7$. Fahr des Hizkia"

,Fahr der Eroberung Samarias $=8$. Fahr des Hizkia". 


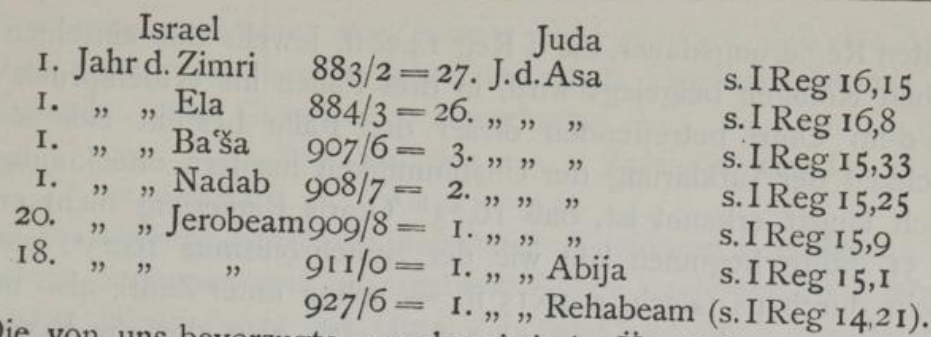

Die von uns bevorzugte "synchronistische Überlieferung" bietet hiermit eine Liste, für die ein dreifaches charakteristisch ist: die für jede israelitische und jüdische Chronologie geltende Bedingung, Jerobeams und Rehabeams Regierungsantritt mehr oder weniger zusammenfallen zu lassen, erfüllt sie in hinreichendem Maße, von den absoluten Zahlen, mit denen I Reg I 5,2 ff. die Regierungsdauer der jüdischen Könige bestimmen, weicht sie nicht $\mathrm{ab}^{\mathrm{T}}$ und steht dennoch andererseits mit der

1 Dies gilt auch für Jehošafat, dem von der obigen Liste 22 Jahre, von I Reg 22,42 dagegen 25 Jahre zugesprochen werden. Denn diese Divergenz, bei der schon die oben behandelten Analogien in den Zeiten des Amașja und seiner 3 Nachfolger an eine Usurpation oder Regentschaft des Jehoram denken lassen, der ungeachtet die Zählung nach Jahren Jehošafaț fortgesetzt worden wäre, wird durch die Nachricht erklärt, mit der II Chr 2I,I ff. das Königsbuch wie folgt ergänzt: ,und Jehošafat legte sich zu seinen Vätern und wurde bei seinen Vätern in der Stadt Dawids begraben und König wurde sein Sohn JehoZecharjahu, Azarjahu. Der hatte Brüder, die Söhne Jehošafaț, nämlich Azarja, Jehiel, von Israel (sic). Und und Kleinodien Jehoram gegeben; festen Städten in Juda; aber das Königtum hatte er dem gegen die Könicherene. Und Jehoram erhob sich wurde souverain, vgl. insbesondere auch von den Fürsten Israels. regierte er zu Jerusalem ... Jönig wurde und 8 Jahre Mitregenten erhoben, we... Jehošafạt hat also Jehoram noch zu seinen Lebzeiten zum Unbeliebtheit II Chr wates er diesem Schwiegersohn des Ahab, von dessen gemäß ist auch II Reg 8, I 6 imten weiß, die Nachfolge sichern wollte. (Dem Joram b. Ahab = Antrittsjahr des Jeho Recht, wenn es den Synchronismus „5. Jahr des melek Jehūdā „während Jüch den Zustan dssatz wīhošăfat zum Mitregenten " vom Thron ges to

Andererseits hält es freilich Brüder beseitigt, s. II Chr 2I,4 und vgl. noch unten S. 24 Sturz gestorben sei; denn der Liste unten S. 25 ergibt, seinem Hause bereiteten, was ganz überlebt, welche Jehu und 'Ațalja $\mathrm{da} ß$ Jehošafat seine Entthon ram zu seinen so er selbst als Mitregent, sondern als Regent, ausas Lebzeiten zu herrschen begann, allerdings nicht 39. Jahre schwer erkrante. (I922) S. 158 hervorgehen erhalten (z. T. gegen Kugler a, a. O.): 
absoluten Regierungsdauer, die I Reg I4,20ff. jeweils den einzelnen israelitischen Königen beigelegt wird, in drei Fällen im Widerspruch ${ }^{x}$.

In dem 'Omri betreffenden dieser drei Fälle besteht eine sichere Möglichkeit der Aufklärung der Unstimmigkeit insofern offenkundig und ja auch längst erkannt ist, daß $16,23^{\mathrm{b}}$ 'Omris Regierung nicht erst in Asas 31. Jahre beginnen läßt wie der Synchronismus $16,23^{2}$, sondern - nicht durchaus falsch, s. I6,15 ff. - schon unter Zimri, also bereits im 27. Jahre des Asa. In $16,23^{\text {b }}$ äußert sich also das die Rechnung nach Königsjahren erfahrungsgemäß stets beeinflussende Bestreben aller Usurpatoren, ihre Regierungsjahre vom Augenblick ihrer ersten Erhebung an auch dann zu zählen, wenn die Empörung erst nach Jahren zum Ziele führt. Die absolute Zahl in $16,23^{\mathrm{b}}$, die 'Omri 12 Jahre gibt, mußs mithin aus einer 'Omri nahestehenden, vermutlich israelitischen Quelle geflossen sein, der Synchronismus $16,23^{a}$ dagegen aus einer 'Omri feindlich oder mindestens gleichgültiger gegenüberstehenden Quelle, die sehr wohl jüdisch gewesen sein kann. ${ }^{2}$

Wer indessen diese Eigenart jeder der beiden Zeitangaben von I Reg 16,23 verkennend meinte, die 12 Jahre 'Omris hätten erst im 3I. Jahre des Asa begonnen, und auf der anderen Seite auch ohne Benutzung israelitisch-jüdischer Synchronismen der Zeit 'Omris und Ahabs, etwa mit Hilfe einer jüdischen Königsliste, noch zu bestimmen vermochte, in welchem Jahre - etwa seit der Gründung des Tempels oder auch nur einer nach den Jahren Asas zählenden Ära - Jehošafaț

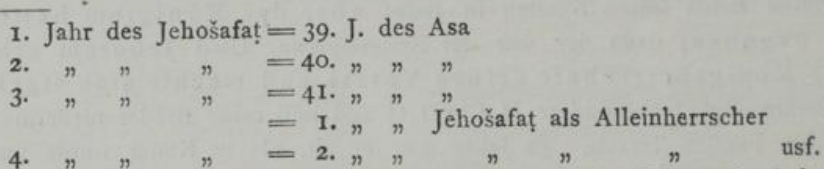

Zugunsten dieser Auffassung spricht ferner noch der wichtige Umstand, daß die II Chr

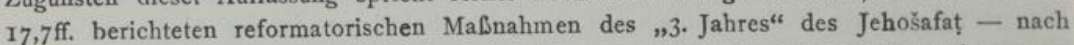
unserer Auffassung also des Todesjahres des Asa - von hier aus den Charakter der ersten Regierungshandlung eines neuen Herrschers erhalten, der sogleich bei der Übernahme der Regierungsgewalt seine fromme Gesinnung bekunden will. Auf die gleiche Weise hat sich denn später auch Hizkija der Priesterschaft empfohlen, indem er gleichfalls in dem Augenblicke, in dem er souverain wurde, bekundete, wie er im Gegensatze zum bisherigen Regime zu handeln beabsichtige, vgl. II Chr 29,3 ff. und im übrigen oben S. 193.

Möglich, aber andererseits nicht beweisbar ist schließlich, daß auch die Erhöhung der 20 Jahre Ahabs auf 22 Jahre, die $16,29^{\mathrm{b}}$ vorliegt (s. sofort), von hier aus ihre Erklärung findet; denn wer Jehošafat statt 22 faktischer Regierungsjahre 24 Jahre zuwies, weil er seine beiden Regentschaftsjahre nicht mehr als solche erkannte und rechnete, kann angesichts der chronologischen Unstimmigkeiten, die er hierdurch herbeiführte und die er doch wieder ausgleichen mußte, sehr wohl auch Jehošafaț israelitischem Zeitgenossen 2 Jahre zugelegt haben.

I Nämlich bei Jerobeam I., 'Omri u. Ahab, denen sie 2I, 8 u. 20 Jahre zuweist an Stelle der 22,12 u. 22 Jahre, die I Reg 14,20; 16,23 und 16,29 angeben.

2 Vgl. hierzu auch die theoretischen Ausführungen unten S. $29 \mathrm{f}$, die von anderen Gesichtspunkten aus zu einem ganz ähnlichen Ergebnis führen. 
zur Regierung gekommen war, konnte nicht glauben, daß das Antrittsjahr Jehošafațs mit dem 4. Jahre des Ahab identisch sei wie I Reg 22,4I angibt. Auf Grund einer Berechnung

$$
\begin{aligned}
& \text { 31. Fahr des Asa }=1 . \text { Fahr des 'Omri } \\
& 4 I \text {. (letztes) }, ", \quad, \quad, \quad, \quad, \quad
\end{aligned}
$$

mußte er vielmehr annehmen, Jehošafaț Regierung habe im II. Jahre des 'Omri begonnen und entsprechend Ahabs Regierung im 2. Jahre des Jehošafat. Wenn daher der 'Omri, Jehošafat und Ahab gewidmete Abschnitt der LXX (III Reg I6,23 ff. und zwar besonders 16,28 ff.) dem Ergebnis des soeben entwickelten Gedankenganges genau entsprechend vom Masoretischen Text tatsächlich in den Gleichungen

$$
\begin{aligned}
& \text { II. Fahr des 'Omri }=1 \text {. Fahr des Fehošafat } \\
& \text { 2. ", Fehošafat }=1 . \quad, \quad \text { "Ahab }
\end{aligned}
$$

abweicht, so zeigt das von neuem, daß erst die Hinzufügung, Abrundung oder spätere unzutreffende Interpretation der absoluten $\mathrm{Re}$ gierungszahlen zu Unstimmigkeiten geführt hat. Denn die Möglichkeit, die Widersprüche in den Einleitungsformeln und die Schwankungen der Überlieferung umgekehrt etwa auf fehlerhafte sekundäre Hinzufügung der Synchronismen zurückzuführen, besteht ja auch hier deshalb nicht, weil, selbst wenn man die unbedingte Richtigkeit der für Asa und 'Omri überlieferten absoluten Regierungszahlen und somit auch der Gleichung 11. Fahr des 'Omri = I. Fahr des Fehošafat

voraussetzen würde, immer noch unerklärlich bliebe, warum der Masoretische Text (I Reg 22,41) und ebenso bekanntlich auch die LXX (III Reg 22,4I) auch noch den Synchronismus 4. Jahr des Ahab = I. Jahr des Jehošafat
bieten, mithin in einer Variante zu LXX (III Reg $16,28^{a}$ ) den Antritt
des Jehošafat um 4 Jahre verzögern.

Aber nicht nur Synchronismen und ,Jüdische Königsliste“, wie wir die für die jüdischen Könige überlieferten absoluten Zahlen der jeweiligen Regierungsdauer auch hier kurz nennen wollen, zeugen hier wie sonst gemeinsam gegen die Zuverlässigkeit bezw. die Ursprünglichkeit der überlieferten absoluten Regierungszahlen der israelitischen Könige; vielmehr erweckt auch die äußere Form, in der diese Zahlen mitgeteilt werden, mehrfach Bedenken: bei den jüdischen Königen dient der Mitteilung der absoluten Regierungsdauer regelmäßig die breite Formel ,x Jahre war er alt, als er König wurde und y Jahre regierte er in Jerusalem und seine Mutter hieß NN aus Z.", bei den Israeliten dagegen folgt die absolute Jahreszahl der Synchronismus-Formel, welche bei israelitischen und jüdischen Königen bekanntlich unverändert bleibt, meist als ganz knapper Zusatz „y Jahre“, so daß auch die äußere Gestalt der „Einleitungsformeln" vermuten läßt, daß — im Gegensatz 
zu den Synchronismen - die absoluten Jahreszahlen mindestens bei den israelitischen Königen Zusätze oder vielmehr planmäßige nachträgliche Ergänzungen sind.

Darf und muß bereits aus diesen Gründen die Autorität der absoluten Regierungszahlen, die I Reg $16,23 \mathrm{ff}$. für 'Omri und Ahab geben, als gering erachtet werden, so gilt dies von der für Jehoram b. Ahab II Reg 3,I überlieferten absoluten Zahl von 12 Jahren, mittels derer wir die oben S. 2of. gegebene Synchronismenliste an das Jahr 84I/0 versuchsweise anknüpten, in ganz besonderem Maße; denn die assyrischen Angaben über Ahab und die Schlacht bei Qarqar sind ja mit der Gleichung „letztes Regierungsjahr des Ahab $=853 / 2$ " schlechthin unvereinbar: nach Salmanassars bekannten Berichten hat Ahab im Jahre 853 Damaskus im Kampfe gegen Assyrien tatkräftig unterstützt; in diesem Jahre bestand also ein Bundesverhältnis zwischen Damaskus und Israel. Und im Einklang damit weiß ja bekanntlich auch die Bibel (I Reg 20,3 I ff.) von einem Vertrage zwischen Ahab und dem König von Aram, dessen Bruch durch $\mathrm{Ahab}$ und seinen jüdischen Bundesgenossen Jehošafat erst nach einer zweijährigen Ruhezeit in einem "dritten Jahre“" erneuten Krieg mit den Aramäern und Ahabs Niederlage und Tod bei Ramot-Gil ad zur Folge hatte.

Sind aber Salmanassars Angaben über die Namen der Verbündeten, die ihm bei Qarqar gegenüberstanden, und der alttestamentliche Bericht über Ahabs Ende a priori ebenso glaubwürdig wie umgekehrt die alttestamentliche Angabe über die absolute Regierungsdauer des Jehoram aus den oben genannten Gründen von vornherein für nicht ganz sicher gelten muß, so entfällt damit jede Möglichkeit, dem von Abab durch Ahazja getrennten Jehoram die Jahre $852-84 \mathrm{I}$ zuzuweisen. Mit anderen Worten: die II Reg 3,I angegebene absolute Zahl der Regierungsdauer des Jehoram b. Ahab darf zur Rekonstruktion der Chronologie der israelitischen und jüdischen Könige von Salomo bis Jehoram b. Ahab bezw. Ahazja b. Jehoram b. Jehošafaț nicht herangezogen werden, vielmehr muß die Anknüpfung dieser älteren Epoche an die mit Jehus Usurpation beginnende zweite in anderer Weise und zwar so erfolgen, daß Jehoram b. Ahab höchstens ro Jahre zufallen. ${ }^{\mathrm{T}}$ Demgemäß ziehen wir nunmehr zwar wiederum die vor Jehorams sechstem Jahre liegenden Synchronismen heran, verwerten daneben aber auch die mit einer Ausnahme bisher stets bewährte "Jüdische Königsliste" und die bereits oben S. $2 \mathrm{I}^{\mathrm{x}}$ zitierte Zusatzquelle II Chron $2 \mathrm{I}, 2-4$ und erhalten dadurch folgende Ergänzungen bezw. Verbesserungen der oben S. 2of. versuchsweise aufgestellten Liste:

I So nach der approximativen Berechnung: Schlacht bei Qarqar $=853$; Ahabs Tod frühestens im 3. Jahre nach dieser $=85 \mathrm{I}$, Ahazjas Tod im 2. Jahre nach diesem $=850$, Jehorams Ende durch Jehu $84 \mathrm{r} / \mathrm{o}$. 
bei den achträg.

der abb geben, b. Abyab derer wir II/O ver. yrischen mit der $n$ unver. hre 853 diesem IIsrael. 0,31 ffi.) Bruch heiner n Arahatte. deten, Bericht ie althoram sicher hazja

prten:

des

raeli-

bezw.

mehr

ation

ram

nun-

Syn-

Aus:

reits

urch

chis:

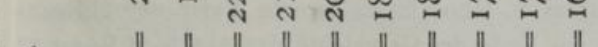

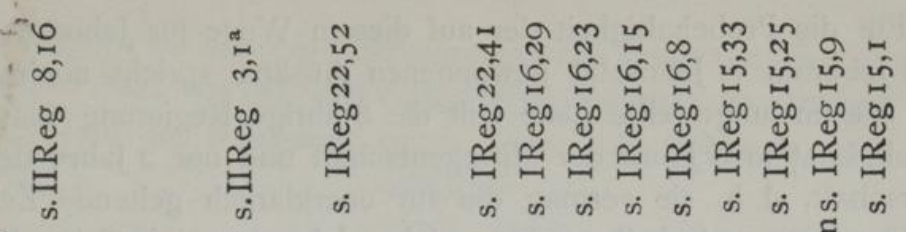

हี

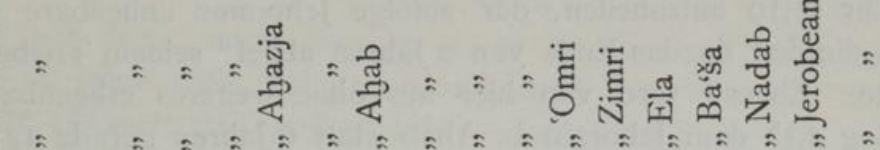

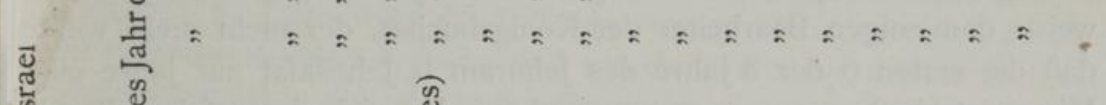

营 $\quad$ 营 $=$

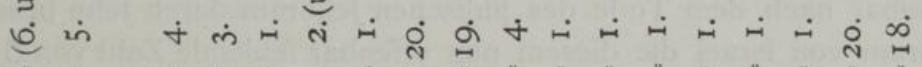

$\begin{array}{llllllllllllllllll} & \| & \| & \| & \| & \| & \| & \| & \| & \| & \| & \| & \| & \| & \| & \| & \| & \|\end{array}$

竎

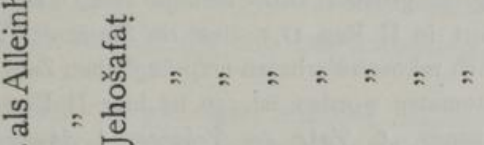

$\frac{\vec{y}}{\tilde{y}}=\vec{y}=====$

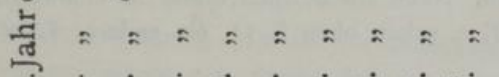

सं

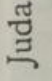

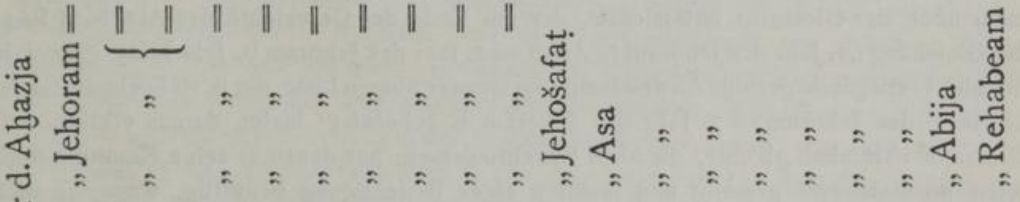

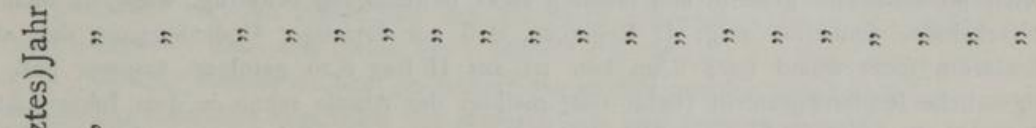

赵

¿̇ :

- $\dot{\alpha}$ ம

$\begin{array}{lllllllllllllllllllllllll} & \| & \| & \| & \| & \| & \| & \| & \| & \| & \| & \| & \| & \| & \| & \| & \| & || & \| & \mid & \|\end{array}$

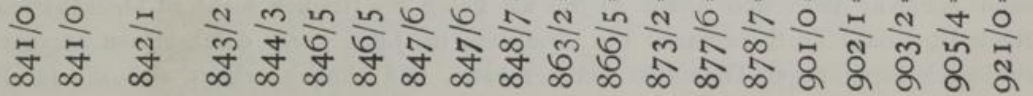


Für die Probehaltigkeit der auf diesem Wege für Jehoram b. Ahab und Jehoram b. Jehošafat gewonnenen Ansätze spricht noch anderes: die soeben aufgestellte Liste teilt die 8 jährige Regierung des Jehoram b. Jehošafat in 6 Jahre der Mitregentschaft und nur 2 Jahre der Alleinherrschaft, d. h. sie vermag die für unerklärlich geltende Zeitangabe II Chr 21,19 aufzuhellen, der zufolge Jehorams unheilbare Krankheit „um die Zeit da das Ende von 2 Jahren ablief" seinem Treiben ein Ziel setzte. Ebenso wird von hier aus ohne weiteres erkennbar, warum II Reg $3, \mathrm{I}^{\mathrm{b}}$ dem Jehoram b. Ahab statt 6 Jahren gerade 12 Jahre zuweist: demjenigen Bearbeiter des Königsbuches, der nicht mehr wußte, daß die ersten 6 der 8 Jahre des Jehoram b. Jehošafat nur Jahre einer Mitregentschaft gewesen waren, und für den sich demzufolge die Gesamtsumme der der 'Ațalja vorangegangenen jüdischen Könige um 6 Jahre erhöhte, konnte es sehr wohl notwendig scheinen, dem fast unmittelbar nach dem Tode des jüdischen Jehoram durch Jehu beseitigten Jehoram von Israel die diesem nun offenbar fehlende Zahl von 6 Jahren zuzulegen. ${ }^{x}$

I Diese Korrektur ähnelt in hohem Maße derjenigen, die, wie wir oben S. 15 ff. sahen, II Reg 15,27 erfahren hat. Auch das ist den beiden Fällen irriger Korrekturen gemeinsam, daß̧ die Verlängerung der absoluten Regierungsdauer eines Königs einen nachfolgenden Synchronismus mitbetroffen hat. Wie dort in II Reg 17,1 eine im Sinne der "Änderung ja durchaus folgerichtige Erhöhung der noch rekonstruierbaren ursprünglichen Zahl ,9. Fahr des Ahas" auf 12. Jahr des Ahaz vorgenommen worden ist, so ist hier II Reg 8,25 der 2u postulierende ursprüngliche Synchronismus „6. Fahr des fehoram b. Ahab = . Fahr des Ahazja" durch die zwar folgerichtige, aber irrige Gleichung „12. Jahr des Jehoram = I. Jahr des Ahazja" verdrängt worden. Noch ein Beispiel dieser so charakteristischen Arbeitsweise bietet II Reg 12,2 und 13,I, s. schon oben S. I1, ein anderes LXX III Reg 16,23 ff., s. oben S. 22 f.

Über das wahre Verhältnis des Jehoram b. Jehošafat zu seinem Vater war übrigens auch noch der Glossator unterrichtet, der am Ende der Geschichte des Ahazja II Reg 1,17 die Gleichung „1. Jahr des Jehoram b. A hab = 2. Jahr des Jehoram b. Jehošafaț “ gibt. Ob sich die hier vorliegende geringe Abweichung von unserer obigen Liste, die ja vielmehr die Gleichung "t. Jahr des Jehoram = 3. Jahr des Jehoram b. Jehošafaț" bietet, daraus erklärt, daß der Glossator irrtümlich glaubte, die alten Synchronismen, aus denen er seine Kenntnis schöpfte, seien postdatierend gemeint und insofern einer Berichtigung bedürftig, wage ich nicht zu entscheiden. Immerhin zeigt II Reg 9,29, daß ein derartiger Gedankengang den alten Erklärern nicht fremd war; denn hier ist aus II Reg 8,26 gefolgert worden, daß der eigentliche Regierungsantritt (hebr. rê̌īt malkūt funden haben müsse, das dem 8,25 - wie wir sahen, irrtümlich - genannten „I2. Jahre“ voranging.

Bemerkenswert und vielleicht im Sinne einer weiteren Bestätigung unserer obigen Ansätze verwertbar ist schließlich noch der Umstand, daß die Kämpfe Ahabs und Jehorams um Ramot-Gil'ad unserer Liste zufolge in den Jahren $847 / 6$ und $841 / 0$ stattgefunden haben; denn nunmehr stellt sich Ahabs Angriff als Folge der (freilich nicht zu überschätzenden) Niederlage dar, die Salmanassar den Aramäern im Jahre zuvor durch seinen sogenannten zweiten Feldzug gegen Damaskus bereitet hatte, und ähnlich erklärt sich die für Israel 
Verlängern wir die obige ,synchronistische Liste“ um die I Reg I I,42 überlieferte Zahl von 40 Regierungsjahren Salomos, so erhalten wir

I. Jahr des Rehabeam 921/0 = 40. Jahr Salomos

$$
\begin{aligned}
& 960 / 59=1 . " \quad " \quad \\
& 957 / 6=4 . \quad \text { (s. IReg } 6,1 ; 37) .
\end{aligned}
$$

Hiernach bezw. nach dem Jer 52,12 gegebenen Datum der Zerstörung im Jahre $586 / 5$ hat der salomonische Tempel etwas länger als 370 Jahre 4 Monate Io Tage bestanden - Josephus (X I47) gibt 470 Jahre 6 Monate ro Tage, trotz der Differenz von genau hundert Jahren $\mathrm{m}$. E. ein weiteres Anzeichen dafür, daß wir dank der assyrisch-israelitischen Synchronismen in den obigen Ausführungen die ursprünglichen Angaben der synchronistischen Überlieferung zu erschließen und richtig zu interpretieren vermochten. ${ }^{x}$

\section{VII}

Schon die enge Verwandtschaft zwischen den Zeitangaben der „Babylonischen Chronik“ und den sogenannten Einleitungsformeln der einzelnen Kapitel des Königsbuches ließ uns a priori vermuten, daß die israelitisch-jüdischen Synchronismen wenigstens ebenso alt und glaubwürdig`seien als die sie begleitenden absoluten Zahlen. Darüber hinaus

offensichtlich viel günstigere Situation, während derer Jehu den Jehoram beseitigte, jetzt als unmittelbare Folge der Feldzüge, die Salmanassar 845 und $84 \mathrm{I}$ unternahm und die die Assyrer zuletzt $\left(84 \mathrm{I}\right.$, s. oben S. $\mathrm{IO}^{2}$ ) bekanntlich bis vor Damaskus und in den Hauran führten.

I Welcher Autorität sich die mir unbekannte Quelle erfreute, der Josephus seine Angabe entnahm, erhellt $u$. a. daraus, daß er nach ihr die gesamte Regierungszeit des Dawidischen Königshauses auf 514 Jahre 6 Monate 10 Tage berechnet hat ( $\$ 143)$, obwohl diese Zahl seinen vorangegangenen Angaben über die Regierungsdauer der einzelnen Könige, in denen er im allgemeinen den absoluten Zahlen des Königsbuches folgt, glatt widerspricht.

Im übrigen sei wenigstens kurz noch darauf hingewiesen, daß die von Josephus so überraschend herangezogene Tradition, die ja wohl aus Priesterkreisen stammen dürfte, den Monat Ziw (I Reg 6,I; 37, vgl. dazu auch II Chr 3,2) keinesfalls dem Ijar gleichgesetzt haben kann, da sie, die offenkundig das Jer 52,12 mitgeteilte Datum akzeptiert, sonst niemals gerade die Zahl 470 (bezw. 370!) Jahre sechs Monate 1o Tage hätte errechnen können. Vielmehr scheint sie den ${ }^{2}$. Monat" des zu Salomos Zeit gebräuchlich gewesenen kanaanäischen Kalenders dem Šebaț der späteren Zeit gleichgesetzt zu haben, so daß hier. nach der "7. Monat", der "Festmonat Etanïm" (I Reg 8,2), dem babylonischen Tammuz, der "Bul“ genannte „8. Monat" (I Reg 6,38) dem Ab entsprechen würde. Josephus selbst ist freilich auch hier unkritisch verfahren, insofern er den "2. Monat" namens Ziw dem Ijar gleichsetzt (VIII \$ 6I) und die Zerstörung des Tempels (X $\$$ 146) im Gegensatz zu Jer 52,12 am I. Ab stattfinden läßt. Ein Hindernis, den Ziw dem späteren Šebaț mehr oder weniger genau gleichzusetzen, sehe ich übrigens nicht, da im Februar die Hauptregenzeit, die einen Bau ausschließt, bereits zu Ende geht, und der spätere Tammuz ist der gegebene Festmonat des phönizisch-kanaanäischen Gebietes. 
weisen aber auch die „Einleitungsformeln“ des Königsbuches und dessen ganze Komposition Merkmale auf, die den gleichen Schluß empfehlen: Nicht die absoluten Jahreszahlen, sondern die Synchronismen stehen a m Beginn der Formel, mit der zur Regierung jedes neuen Königs übergeleitet wird. ${ }^{x}$ Ohne Rücksicht darauf, ob es sich um einen israelitischen oder um einen jüdischen König handelt, bleibt der Wortlaut der Synchronismus-Formel der gleiche, aber die Form, in der die absoluten Jahreszahlen mitgeteilt werden, richtet sich danach, welchem der beiden Reiche der betreffende König angehört. Wie bereits oben S. 23 erwähnt, folgen sie bei den Israeliten meistens als ganz knapper Zusatz „y Jahre in . ....., bei den Juden dagegen erscheinen sie in der breiten Formel: „x Jahre war er alt, als er König wurde und y Jahre regierte er in Jerusalem und seine Mutter hieß NN aus $Z$ ". Somit lassen auch die äußeren Merkmale der Einleitungsformeln die Annahme zu, daß nicht die absoluten Jahreszahlen, sondern vielmehr die Synchronismen primär sind, in der Weise, daß bei oder auch nach der Komposition des uns überkommenen Königsbuches alten Synchronismen, die für die Könige von Israel vorlagen, mittels einer bereits vorhandenen Quelle oder auch mittels einer zu diesem $Z$ wecke neu berechneten Liste gerade noch die absoluten Regierungszahlen beigefügt wurden, während bei den jüdischen Königen ausführlichere einander ergänzende Angaben ausgéschöpft wurden, welche außer der absoluten Regierungsdauer auch das Alter des Fürsten und den Namen seiner Mutter überlieferten. ${ }^{2}$

Bedeutungsvoller als die Zusammensetzung der "Einleitungsformeln" ist indessen der ,allgemeine Synchronismus der hebräischen Könige“,

I Für Josephus, der zu jüdischen Königen mehrfach nur mit dem Synchronismus, also ohne Beifügung der absoluten Regierungszahl überleitet, vgl, die nächste Anm. am Ende.

2 Mit den Worten ,y Jahre regierte er in Jerusalem" erinnert die bei den jüdischen Königen übliche Formel an die Formel „y Jahre regierte A über Babel (bezw. Elam usw.), sein Sohn B setzte sich in Babel (Elam usw.) auf den Thron", mit welcher die "Babylonische Chronik" den jedem Könige gewidmeten Abschnitt jeweils zu beschließen pflegt (vgl. die Zitate oben S. 7f.). Man darf daher vielleicht vermuten, daß dieser Teil der jüdischen „Einleitungsformeln" auch in der jüdischen Chronik, die für das uns erhaltene Königsbuch exzerpiert wurde, ursprünglich am Schlusse jedes Abschnittes hinter den Worten ,und A legte sich zu seinen Vätern und wurde begraben in ..." gestanden habe und erst sekundär in die „Einleitungsformel“ geraten sei. Ist dieser Analogieschluß erlaubt, so muß man feststellen, daß Josephus die ältere Fassung vorgezogen hat; denn dieser benutzt gern eine im genauen Wortlaute zwar etwas schwankende, im ganzen sich jedoch gleichbleibende Schlußformel „A verschied, nachdem er $\mathrm{x}$ Jahre gelebt und $\mathrm{y}$ Jahre regiert hatte, und wurde in ... bestattet, die Regierung kam an seinen Sohn B“. Dem entspricht, daß er dort, wo er den Übergang zur Regierung eines neuen jüdischen Königs durch einen Synchronismus vollzieht, nur diesen, nicht aber auch die absolute Regierungszahl an den Anfang des betreffenden Abschnittes stellt, während er bei den israelitischen Königen die Einleitungsformel des Königsbuches hat. 
den schon Wellhausen bemerkt und dahin formuliert hat, daß ,sich die Aufzählung nach dem Datum der Thronbesteigung richtet, in der Weise, daß die Könige der beiden Reiche promiscue an die Reihe kommen, und wer zuerst angetreten ist, auch zuerst und zwar vollständig abgehandelt wird. Z. B. ist im ganzen die Regierung Josaphats von Juda mit der Ahabs von Israel gleichzeitig; weil er aber etwas später angetreten ist, wird er, trotz 22, Iff., erst nachgebracht, nachdem Ahab bestattet ist I $22,4 \mathrm{I}-5 \mathrm{I}$, und dann auch gleich abgemacht, obwohl er noch den Ahazja von Israel überlebt hat. In der LXX tritt Josaphat früher als Ahab an, also steht er auch vor jenem; in richtiger Einsicht in das Prinzip dieser Anordnung"." Die herrschende Auffassung vom Unwert der speziellen Synchronismen der „Einleitungsformeln" sollte also konsequenterweise zu dem Schluß führen, daß der Epitomator, der aus einer jüdischen und aus einer israelitischen Chronik den Kern des uns vorliegenden Königsbuches komponiert hat, ein allgemein-synchronistisches Prinzip anwandte, ohne daß aus älterer Zeit auf ihn gekommene spezielle Synchronismen ihm seine eigenartige allgemein-synchronistische Darstellungsweise nahegelegt bezw. ermöglicht hätten. Befriedigt diese notwendige Folgerung um so weniger, weil mit ihr nicht erklärt wird, warum man sich nicht mit dem ,allgemeinen Synchronismus“ begnügte, so ist die geltende Anschauung zudem noch deshalb unwahrscheinlich, weil auch der sogenannte allgemeine Synchronismus keine Eigentümlichkeit jüdischer Geschichtsschreibung ist; denn gleich den speziellen Synchronismen, die, wie wir eingangs sahen, für die „Babylonische Chronik" charakteristisch sind, ist auch der "allgemeine Synchronismus" den Babyloniern und Assyrern vertraut gewesen: deren synchronistische Königslisten weisen z. T. dieselbe Anordnung wie das hebräische Königsbuch auf. ${ }^{2}$ Soll man aber wirklich glauben, daß die Juden ursprünglich nur eine der der altorientalischen Chronistik vertrauten Methoden hätten anwenden wollen? daß sie sich zunächst darauf beschränkt hätten, das Prinzip des allgemeinen Synchronismus zu benutzen, um erst nach Jahrzehnten die speziellen Synchronismen nachzutragen und diese dann auch gleich an die Spitze der „Einleitungsformeln“ zu stellen?

Eher möchte ich umgekehrt vermuten, daß das „Buch der Geschichte der Könige von Juda" und das entsprechende Werk über die

I S. Bleek (-Wellhausen), Einleitung in das A. T. ${ }^{4}$ (1878) S. 243 ; vgl. auch Kamp. hausen, Die Chronologie der hebräischen Könige (1883) S. 49.

2 Obwohl ihm die Analogie des biblischen Königsbuches offenbar nicht gegenwärtig war, hat Ungnad dennoch bereits ZDMG 72 (1918) S. $313 \mathrm{ff}$. überzeugend darzutun vermocht, welches Prinzip auf der Tontafel VAT II 338 (jetzt $=$ KAV Nr. 12), dem winzigen Fragment einer besonders gearteten, zusatzlosen synchronistischen Königsliste aus Assur angewandt worden ist, vgl, noch Weidner MVAG I92I, 2 S. 2 f. 
Könige von Israel im großen und ganzen der „Babylonischen Chronik“ ähnlich waren, trotz gewisser kleinerer Unterschiede sowohl dieser gegenüber wie auch wiederum unter sich, insbesondere darin, daß sie die in den Nachbarstaaten erfolgenden Thronbesteigungen verzeichneten. Erst das Vorhandensein der in diesen Notizen der Einzelchroniken naturgemäß liegenden speziellen Synchronismen wird es dem Bearbeiter, der Exzerpte aus beiden Werken zum Kern des uns allein erhaltenen Königsbuches vereinigte, ermöglicht haben, das Prinzip des allgemeinen Synchronismus anzuwenden, d. h. etwa wie folgt zu verfahren: sobald er aus dem ihm überkommenen Bericht über die Taten eines bestimmten Königs entnommen hatte, was ihm erwähnenswert schien, griff er auf den innerhalb des eben ausgezogenen Abschnittes enthaltenen Synchronismus zurück, indem er diesen als Überleitung zu dem in die gleiche Zeit gehörigen Könige des Nachbarreiches benutzte, und schloß hieran, was er in der anderen der beiden ihm vorliegenden Einzelchroniken über diesen König fand bezw. der Úbernahme in sein neues Werk für wert erachtete. ${ }^{x}$ Mit anderen Worten: der Synchronismus, der die Thronbesteigung eines jüdischen Königs fixiert, dürfte jedesmal aus der „Israelitischen Chronik“ entnommen sein, während die unmittelbar darauf folgenden Angaben über das Alter, die Regierungsdauer, die Mutter und die Taten des betreffenden Königs sowie die Schlußformel auf die "Jüdische Chronik" zurückgehen, und umgekehrt. Diese Vorstellung von den Quellen und der Arbeitsweise des Epitomators gestattet mithin die Überlieferung der speziellen Synchronismen und die Anwendung des Prinzips des allgemeinen Synchronismus ein und demselben Gelehrten zuzuschreiben, demjenigen, der auf den Gedanken kam, die „Israelitische Chronik" und die "Jüdische Chronik" im Auszuge zu einem einzigen Werke zu vereinigen. ${ }^{2}$ Hierüber

I Wie dieses Verfahren in der Praxis gelegentlich ein Versehen zur Folge haben konnte, ist oben S. 18 gezeigt worden.

2 Von beiden Quellenwerken wissen wir viel zu wenig, als daß wir erkennen könnten, welchem der beiden der Bearbeiter dort den Vorzug gab, wo er Widersprüche fand, bezw. ob er einen Ausgleich versuchte oder - wie das gerade in den orientalischen Werken so oft $\mathrm{zu}$ bemerken ist - bewußt darauf verzichtete, d. h. einander widersprechende Angaben seiner Quellen ohne eigene Stellungnahme aneinanderreihte. Widersprüche werden ihm insbesondere vorgelegen haben, falls bereits jedes der beiden Einzelwerke Angaben über die absolute Regierungsdauer der Könige beider Reiche enthalten haben sollte, was durch die Analogie der "Babylonischen Chronik" ja als möglich erwiesen und auch durch die oben S. 28 erwähnte Verschiedenheit der zweiten Bestandteile der "Einleitungsformeln“ nicht ausgeschlossen wird. Die Differenzen zwischen den synchronistischen und den ab. soluten Angaben, die wir bei Jerobeam I., Ahab und Peqah von Israel hinnehmen mußten, ohne wie in den Fällen des 'Omri, Jehoram, Jerobeam II. und Hosea von Israel, des Jehošafat, Jehoram, Jehoaš, Amașja, Azarja, Jotam und Ahaz von Juda die Ursache des 
hinaus festzustellen, ob Bruchstücke der alten Einzelchroniken oder wenigstens eine uns verlorene ältere und stellenweise ausführlichere Fassung des jetzigen Königsbuches Josephus noch zur Verfügung standen und ob eine Untersuchung, die grundsätzlich nicht von der Analogie der alten „Babylonischen Chronik“, sondern von dem um so viele Jahrhunderte jüngeren Josephus ausgeht, wesentlich andere Resultate wie die unsrige ergibt, wird Aufgabe späterer Forschungen sein, die außerhalb der Kompetenz des Assyriologen liegen.

Widerspruches aufdecken und danach korrigieren zu können, können also mindestens zu einem Teile schon vor der Entstehung des Königsbuches bestanden und als unausgleichbar gegolten haben.

Zeittafel: 


\section{$Z$ eitta fel.}

\begin{tabular}{ll} 
& \multicolumn{1}{c}{ Israel } \\
$922-902$ & Jerobeam I. \\
$902-90$ I & Nadab \\
$90 \mathrm{I}-878$ Ba ša \\
$878-877$ Ela \\
$877 \quad$ Zimri \\
$877-873$ Tibni und Omri \\
$873-866$ Omri \\
$866-847$ Ahab \\
$847-846$ Ahazja \\
$846-84$ I Jehoram
\end{tabular}

960-921 Salomo ${ }^{x}$

92 I-905 Rehabeam

905-903 Abija

903-865 Asa

865-863 Jehošafat als Regent

(863 Tod des Asa)

863-842 Jehošafat

848-842 Jehoram als Mitregent des Jehošafat

$842-84$ I Jehoram

(84I Tod des Jehošafaț)

84I Ahazja

$84 \mathrm{I}-8 \mathrm{I} 5 \mathrm{Jehu}$

$815-799$ Jehoahaz

84I-835 'Ațalja)

799-784 Jehoaš

$784-748$ Jerobeam II.

$748-747$ Zecharja

747 Šallum

747-736 Menahem

736-734 Peqahja

734 Peqah

733-724 Hosea

$724-722$ Belagerung Samarias

835-798 Jehoaš

798-785 Amașja

785-758 Azarja

(770 Tod des Amașja)

758-742 Jotam als Regent

742-734 Abaz als Regent

(734 Tod des Azarja)

734-726 Abaz

(728-726 Hizkia als Mitregent?)

726-697 Hizkia

$697-642$ Manasse

642-640 Amon

640-609 Josia

609 Jehoahaz

$609-598$ Jehojaqim

598-597 Jechonja

597-586 Ședekia

I Zwecks besserer Übersicht sind hier die Zahlen ohne Rücksicht auf den Jahresanfang gegeben; statt $960 / 59$ steht also nur 960 , statt $921 / 0$ nur 921 , statt $586 / 5$ nur 586 usf. 


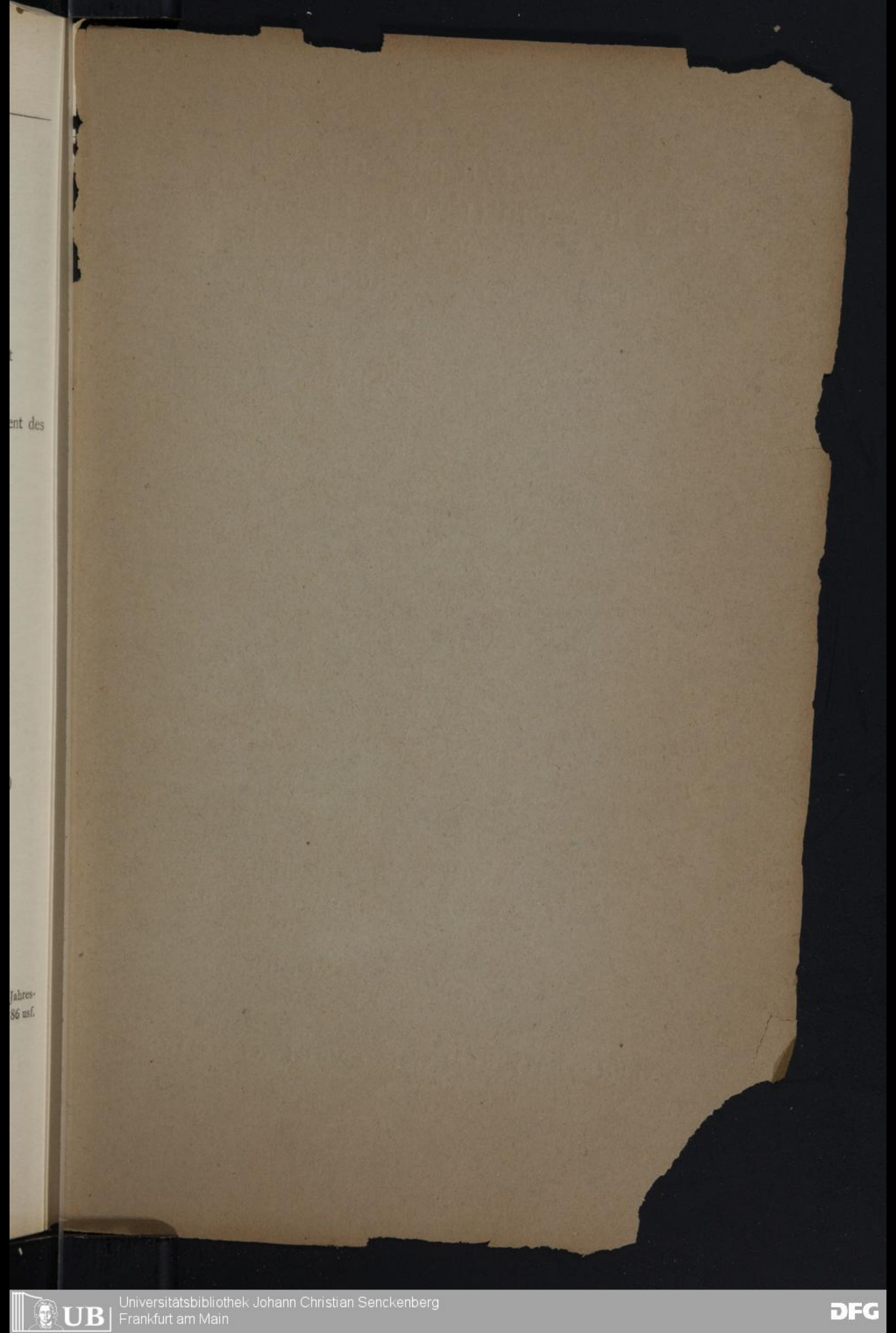


